\title{
DYNAMIC ANALYSES OF A FLEXIBLE VEHICLE MOVING ALONG A FLEXIBLE GUIDEWAY CONSIDERING THE TIP-OFF EFFECT
}

\author{
S. N. CHOU*, F. P. CHENG ${ }^{\dagger}$ and C. S. HUANG* \\ Department of Civil Engineering \\ National Chiao Tung University \\ Hsin-Chu 30050, Taiwan, ROC \\ *snchou48@gmail.com \\ †fpcheng@mail.nctu.edu.tw \\ †cshuang@mail.nctu.edu.tw
}

Received 3 November 2011

Accepted 20 February 2012

Published 6 March 2013

\begin{abstract}
A semi-analytical solution for the tip-off response of a vehicle moving along a guideway is obtained, considering the dynamic interaction between the two subsystems. The guideway is modeled as an inclined simply-supported uniform flexible beam, and the vehicle as a flexible free-free beam under a pre-specified thrust force. The equations of motion for the vehicle and guideway are developed using the Lagrangian approach and the assumed mode method based on the Euler-Bernoulli hypothesis. In the form of nonlinear differential equations, they are solved by the Petzold-Gear backward differentiation formula (BDF) method. The solutions obtained are validated by comparing them with the published results for the models with a rigid vehicle running over a rigid guideway or a flexible guideway. Comparisons of the present solutions with the existing ones for the vehicle and guideway reveal the advantages of the approach proposed herein. Other effects on the tip-off responses of the vehicle that are investigated include the length of the guideway, distance between the shoes of the vehicle, and mass and rigidity ratios of the vehicle to the guideway. The results presented herein provide valuable information for the design of the vehicle launch system.
\end{abstract}

Keywords: Moving load; moving beam model; Lagrangian approach; mode superposition; tip-off responses.

\section{Introduction}

The dynamic response of a beam subjected to a moving vehicle (or structure) has attracted the attention of researchers for a long time. An excellent state-of-art review was given in 1984 by the subcommittee on vibration problems associated with flexural members on transit systems. ${ }^{1}$ The moving vehicle is often modeled as a

${ }^{\dagger}$ Corresponding author. 
moving force, a moving mass, a moving oscillator (also called a sprung mass model) or a moving beam. The moving force model is the simplest and oldest approach, which neglects the interaction between the vehicle and the beam. ${ }^{2-6}$ Research work on this topic can be traced back to 19 th century. ${ }^{2}$ Timoshenko ${ }^{3}$ derived numerous approximate solutions to the problem of a simply-supported beam under the moving loads. Ayre et $a l^{4}{ }^{4}$ studied the transverse vibration of a two-span beam under a moving constant force. The moving force model is known to be valid only for the case when the mass of the moving vehicle is much smaller than that of the beam, and only when the dynamic response of the moving vehicle is not of interest.

A moving mass model is a simple model that to some extent accounts for the interaction between the moving vehicle and the beam. ${ }^{7-11}$ The model was first proposed by Jeffcott ${ }^{7}$ in 1929. Stanišić ${ }^{8}$ employed the Fourier technique to investigate the responses of beams to an arbitrary number of concentrated moving masses. Akin and Mofid ${ }^{9}$ presented a numerical solution by the separation of variables for the dynamic response of an Euler-Bernoulli beam to a moving mass. Their solution scheme is simple and can be used to determine the responses of beams under various boundary conditions. Dehestani et al. ${ }^{10}$ showed that it is necessary to consider the Coriolis acceleration associated with a mass moving along a vibrating beam. $\mathrm{Wu}^{11}$ examined the effects of the inertial, Coriolis, and centrifugal forces induced by noncoupled moving masses on the dynamic responses of an inclined simplysupported beam.

A moving oscillator model includes masses, springs and dampers to capture the dynamic characteristics of a moving vehicle, which is more complicated than a moving mass model. ${ }^{12-15}$ Biggs $^{12}$ presented a semi-analytical solution to the problem of a sprung mass moving on a simply-supported beam. Using a series expansion technique, Pesterev and Bergman ${ }^{13}$ examined the responses of an elastic continuum to multiple moving oscillators. Yang and $\operatorname{Lin}^{14}$ proposed a vehicle-bridge interaction (VBI) element, and Yang and $\mathrm{Wu}^{15}$ further modified the VBI element to investigate the vibrations of simply-supported beams during the passage of high-speed trains.

Unlike a moving oscillator model, which treats a moving vehicle as a discrete system, a moving beam model considers a vehicle as a continuum and particularly as a beam. Cojocaru et $a l .{ }^{16}$ studied the vibration of an elastic bridge under an elastic beam moving at a constant speed. The vehicle was assumed to be connected to the bridge by means of a rigid interface. The quasi-static deformation of the bridge was obtained by the Laplace transform, while the dynamic response of the bridge was determined by the Galerkin method. Zhang and Zheng ${ }^{17}$ investigated the dynamic responses of a simply-supported beam to an elastic beam moving at a constant speed using the modal superposition method. The model consists of two Euler-Bernoulli beams that were connected by flexible springs at two contact points, so that the interaction forces between the two beams are found from the relative deflection of the two contact points. 


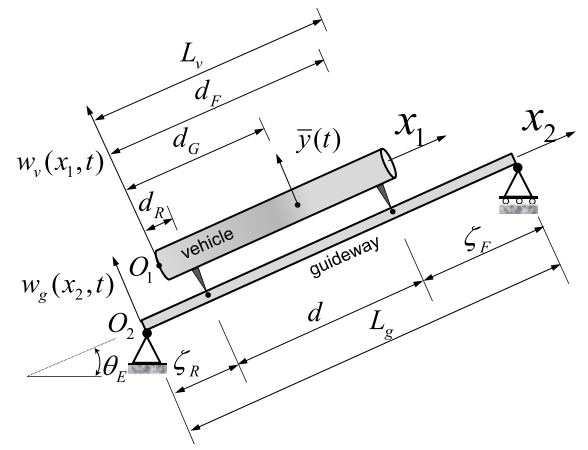

Fig. 1. A typical straight guideway used for vehicle launch.

All the aforementioned studies were focused mainly on the dynamic responses of the beams and are applicable to the design of railroad tracks, railroad bridges and highway bridges. Relatively few studies were focused on the dynamic behavior of the vehicle when it moves along the guideway, which sounds like a missile in a launcher system (see Fig. 1). When a vehicle moves along the guideway, it is mainly subjected to the thrust, inertia and gravity forces, for which two phases can be identified. Before the front shoe of the vehicle (see Fig. 1) loses contact with the guideway, the vehicle is in a two-shoe contact phase. Then the vehicle rotates with respect to its rear shoe when its front shoe loses contact with the guideway. Such an effect is known as tip-off. When the vehicle exhibits tip-off, it is referred to as being "in the tip-off phase". The interaction between the vehicle and its guideway differs considerably between these two phases, and so are the behaviors of the vehicle. Consequently, the dynamic responses of the vehicle in the two phases have to be modeled separately.

In the field of control engineering, generally, a vehicle and its guideway are typically modeled as rigid bodies for the tip-off analysis (Yao and Zhang ${ }^{18}$ ). Although such modeling is quite simple and can be easily used, it does not take into account the dynamic interaction between the vehicle and its guideway. This model fails to yield accurate dynamic responses for the vehicle in real applications when the mass of the vehicle substantially exceeds that of the guideway, and both of the vehicle and guideway are flexible. To overcome this drawback, Chou et al. ${ }^{19}$ proposed a model with a rigid vehicle moving on a flexible guideway. The dynamic responses of the rigid vehicle were indirectly obtained from the guideway responses.

Because the response of the vehicle at take-off significantly affects its flight control, accurately determining the response of the vehicle in the tip-off phase is crucial. In this study, the vehicle and the guideway displayed in Fig. 1 are further modeled as a flexible free-free beam and an inclined elastic simply-supported beam, respectively, based on the Euler-Bernoulli hypothesis. The vehicle is connected to the guideway at two contact points, assumed to be in rigid contact, so that their dynamic responses are the same during the take-off. The equations of motion for the vehicle and guideway, in terms of functions of the configuration coordinates and time, are 
established via the Lagrangian approach with appropriate displacement constraints. A modal superposition technique is adopted to convert the governing equations, in the form of nonlinear partial differential equations, into a set of nonlinear first-order differential equations with time as the independent variable. Then, the Petzold-Gear backward differentiation formula (BDF) numerical method ${ }^{20}$ is employed to solve these first-order differential algebraic equations (DAEs). The solutions obtained are validated by comparison with the published results obtained by models of a rigid vehicle on a rigid guideway or on a flexible guideway. The effects of the guideway length, distance between the vehicle shoes, and mass and flexural rigidity ratios of the vehicle to the guideway upon tip-off of the vehicle are thoroughly studied. The results presented herein provide valuable information for designing the vehicle launch systems.

\section{Theory and Formulation}

Figure 1 shows a straight guideway used for launching a vehicle. While the vehicle moves, the two shoes of the flexible vehicle slide along the elastic guideway by means of a rigid contact. The vector of thrust is assumed to be along the vehicle's centerline (CL) and always coincides with the line joining the two contact points.

\subsection{Position history of vehicle}

The thrust force, $P(t)$ in Fig. 2, acting on the vehicle is predetermined in real applications. Figure 3 shows a typical thrust-time diagram, where $t_{b}$ is the thrust build-up time; $P_{\max }$ is the value of $P(t)$ after time $t_{b} ; t_{F}$ and $t_{R}$ are the times when the vehicle front and rear shoes lose contact with the guideway, respectively. The term $t_{R}$ is called the tip-off time. Between $t_{F}$ and $t_{R}$, the vehicle tip-off occurs.

As mentioned in Sec. 1, two phases exist with the vehicle during take-off, i.e. the two-shoe contact phase and the tip-off phase. From the typical thrust-time curve

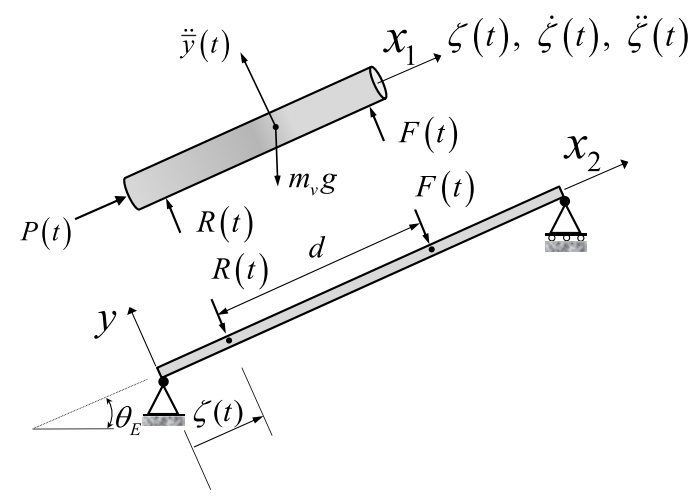

Fig. 2. Free-body diagrams of the vehicle and guideway. 


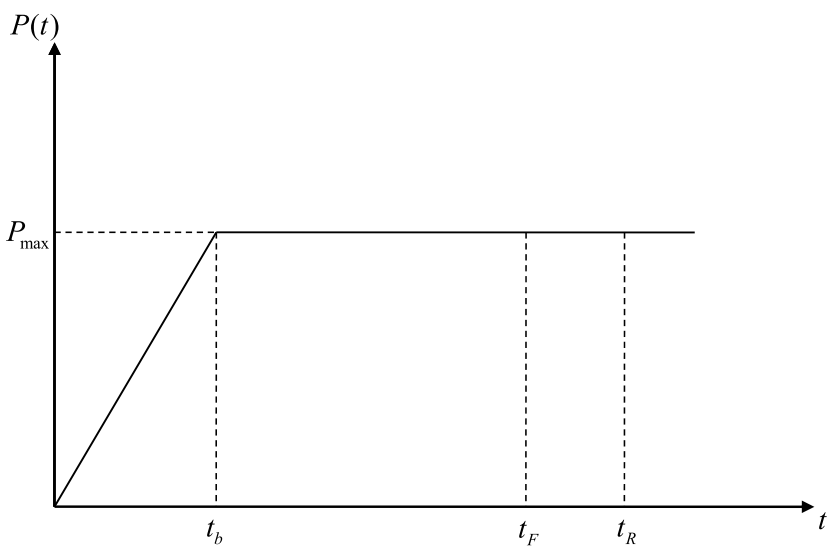

Fig. 3. A typical thrust-time curve.

shown in Fig. 3 and the design parameters of the vehicle and its guideway, one can easily find the position of the rear shoe, $\zeta(t)$ (see Fig. 2), $t_{F}$ and $t_{R}$ can be easily determined to identify the particular phase with which the vehicle is associated at each instant. The formulas for $\zeta(t), t_{F}$ and $t_{R}$ are given below,

- When $0 \leq t \leq t_{b}$ :

$$
\zeta(t)=\frac{1}{m_{v}}\left(\frac{P_{\max }}{6 t_{b}} t^{3}-\frac{1}{2} m_{v} g \sin \theta_{E} \cdot t^{2}\right)+\dot{\zeta}(0) \cdot t+\zeta_{R},
$$

where $m_{v}$ is the mass of the vehicle; $\theta_{E}$ is the angle of inclination of the guideway; $\dot{\zeta}(0)$ is the initial velocity of the vehicle and $\zeta_{R}$ (see Fig. 1) is the distance from the rear shoe of the vehicle to the left end of the guideway when the system is initially at rest (i.e. $\dot{\zeta}(0)=0$ and $\left.\zeta(0)=\zeta_{R}\right)$.

- When $t_{b}<t \leq t_{F}$ :

$$
\zeta(t)=\frac{1}{2 m_{v}}\left(P_{\max }-m_{v} g \sin \theta_{E}\right) \cdot\left(t-t_{b}\right)^{2}+\dot{\zeta}\left(t_{b}\right)\left(t-t_{b}\right)+\zeta\left(t_{b}\right),
$$

where $\dot{\zeta}\left(t_{b}\right)$ and $\zeta\left(t_{b}\right)$ are the velocity of motion and the distance traveled at $t=t_{b}$, respectively, and are determined from Eq. (2.1).

- When $t_{F}<t \leq t_{R}$ :

$$
\begin{aligned}
\zeta(t)=\frac{1}{2 m_{v}}\left(P_{\max }-m_{v} g \sin \theta_{E}\right) \cdot\left(t-t_{b}\right)^{2}+\dot{\zeta}\left(t_{b}\right) \cdot\left(t-t_{b}\right)+\zeta\left(t_{b}\right), \\
t_{F}=t_{b}+\frac{m_{v}}{\left(P_{\max }-m_{v} g \sin \theta_{E}\right)} \\
\cdot\left[\sqrt{\dot{\zeta}\left(t_{b}\right)^{2}+\frac{2}{m_{v}}\left[\zeta_{F}-\zeta\left(t_{b}\right)\right]\left(P_{\max }-m_{v} g \sin \theta_{E}\right)}-\dot{\zeta}\left(t_{b}\right)\right],
\end{aligned}
$$




$$
\begin{aligned}
& t_{R}= t_{b}+\frac{m_{v}}{\left(P_{\max }-m_{v} g \sin \theta_{E}\right)} \\
& \cdot\left[\sqrt{\dot{\zeta}\left(t_{b}\right)^{2}+\frac{2}{m_{v}}\left[\zeta_{F}-\zeta\left(t_{b}\right)+d\right]\left(P_{\max }-m_{v} g \sin \theta_{E}\right)}-\dot{\zeta}\left(t_{b}\right)\right],
\end{aligned}
$$

where $\zeta_{F}$ is the distance from the front shoe of the vehicle to the right end of the guideway at $t=0$, and $d$ is the distance between the two shoes of the vehicle. With the above equations, the values of $\zeta(t), \dot{\zeta}(t)$ and $\ddot{\zeta}(t)$ during the vehicle take-off can be calculated.

\subsection{Two-shoe contact phase}

The dynamic response of the vehicle can be split into two parts, i.e. the elastic deformation and rigid body motion, known to be completely uncoupled. The equations of motion and relevant boundary conditions can be derived using the Lagrangian approach. The kinetic energy and potential energy of the vehicle and the guideway are

$$
\begin{aligned}
K_{v}= & \frac{1}{2} \int_{0}^{L_{v}} \rho_{v} A_{v}\left(\dot{w}_{v}+\dot{\zeta} w_{v}^{\prime}\right)^{2} d x_{1}+\frac{1}{2} m_{v}\left(\dot{\bar{x}}_{r}^{2}+\dot{\bar{y}}_{r}^{2}\right)+\frac{1}{2} J \dot{\bar{\theta}}_{r}^{2} \\
K_{g}= & \frac{1}{2} \int_{0}^{L_{g}} \rho_{g} A_{g} \dot{w}_{g}^{2} d x_{2}, \\
V_{v}= & \frac{1}{2} \int_{0}^{L_{v}}\left(E_{v} I_{v} w_{v}^{\prime \prime 2}-P w_{v}^{2}\right) d x_{1}+\int_{0}^{L_{v}} \rho_{v} A_{v} g \cos \theta_{E} w_{v} d x_{1} \\
& +\left(m_{v} g \sin \theta_{E}\right) \bar{x}_{r}+\left(m_{v} g \cos \theta_{E}\right) \bar{y}_{r} \\
V_{g}= & \frac{1}{2} \int_{0}^{L_{g}} E_{g} I_{g} w_{g}^{\prime \prime 2} d x_{2},
\end{aligned}
$$

where subscripts $v$ and $g$ refer to the vehicle and the guideway, respectively; the overhead dot $(\cdot)$ and the prime (') denote differentiation with respect to time $t$ and coordinate $x$, respectively; $K$ and $V$ are the kinetic energy and potential energy, respectively; $E I$ is the flexural rigidity; $\rho A$ represents the mass per unit length; $J$ is the mass moment of inertia of the vehicle; $\dot{\zeta}(t)$ denotes the velocity of the vehicle in the local $x_{2}$-direction; $\bar{x}_{r}(t)$ is the axial coordinate of the vehicle under rigid body motion in the fixed coordinate system $x_{2} \mathrm{O}_{2} y_{2} ; \bar{y}_{r}(t)$ is the transverse displacement of the vehicle under rigid body motion, and $\bar{\theta}_{r}(t)$ is the angle of rotation of the vehicle under rigid body motion. The transverse elastic displacements of the vehicle $w_{v}\left(x_{1}, t\right)$ and the guideway $w_{g}\left(x_{2}, t\right)$ are described as functions of the axial coordinates $x_{1}$ and $x_{2}$, respectively. 
The two shoes of the vehicle are assumed to slide along the elastic guideway by means of a rigid contact. Hence, the corresponding constraint equations are

$$
\begin{aligned}
& w\left(d_{R}, t\right)=w_{g}(\zeta, t), \\
& w\left(d_{F}, t\right)=w_{g}(\zeta+d, t),
\end{aligned}
$$

where $d_{R}$ is the distance between the left end and rear shoe of the vehicle; $d_{F}$ is the distance between the left end and front shoe of vehicle, and $w\left(d_{R}, t\right)$ and $w\left(d_{F}, t\right)$ are the total transverse displacements at the rear and front shoes of the vehicle, respectively. The displacements $w\left(d_{R}, t\right)$ and $w\left(d_{F}, t\right)$ comprise a rigid part and an elastic part.

The transverse elastic displacements of the beams can be expressed in terms of their normal modes as

$$
\begin{aligned}
& w_{v}\left(x_{1}, t\right)=\sum_{j=1}^{N} \phi_{j}\left(x_{1}\right) Y_{j}^{v}(t), \\
& w_{g}\left(x_{2}, t\right)=\sum_{j=1}^{N} \psi_{j}\left(x_{2}\right) Y_{j}^{g}(t),
\end{aligned}
$$

where $Y_{j}^{v}(t)$ and $Y_{j}^{g}(t)$ are the generalized coordinates corresponding to the $j$ th mode of the vehicle and guideway, respectively; $\phi_{j}\left(x_{1}\right)$ and $\psi_{j}\left(x_{2}\right)$ denote the $j$ th mode shape functions of the vehicle and guideway, respectively.

The vehicle is modeled as a beam with two free ends, and its mode shape functions $\phi_{j}\left(x_{1}\right) \operatorname{are}^{21}$

$$
\phi_{j}\left(x_{1}\right)=\cos \left(\beta_{v j} x_{1}\right)+\cosh \left(\beta_{v j} x_{1}\right)-\Upsilon_{j}\left[\sin \left(\beta_{v j} x_{1}\right)+\sinh \left(\beta_{v j} x_{1}\right)\right],
$$

where $j=1,2, \ldots, N$

$$
\beta_{v j}^{4}=\omega_{v j}^{2} \cdot \frac{\rho_{v} A_{v}}{E_{v} I_{v}}, \quad \Upsilon_{j}=\frac{\cos \left(\beta_{v j} L_{v}\right)-\cosh \left(\beta_{v j} L_{v}\right)}{\sin \left(\beta_{v j} L_{v}\right)-\sinh \left(\beta_{v j} L_{v}\right)}, \quad \beta_{v j} L_{v} \approx\left(j+\frac{1}{2}\right) \pi,
$$

$\omega_{v j}$ is the circular frequency of the $j$ th mode of the vehicle, and $L_{v}$ is the length of the vehicle. The guideway is modeled as a simply-supported beam with the following mode shape functions:

$$
\psi_{j}\left(x_{2}\right)=\sin \left(\frac{j \pi x_{2}}{L_{g}}\right), \quad j=1,2, \ldots, N,
$$

where $L_{g}$ is the length of the guideway.

The motions of vehicle and guideway have to satisfy Lagrange's equations,

$$
\frac{d}{d t}\left[\frac{\partial \mathcal{L}(\mathbf{q}, \dot{\mathbf{q}}, t)}{\partial \dot{q}_{k}}\right]-\frac{\partial \mathcal{L}(\mathbf{q}, \dot{\mathbf{q}}, t)}{\partial q_{k}}=0, \quad k=1,2, \ldots, N,
$$

where the functional $\mathcal{L}$, expressed in terms of the generalized coordinates $q_{k}$ and velocities $\dot{q}_{k}$, represents the difference between the kinetic energy and potential 
energy of a conservative dynamic system. To account for the displacement constraints in Eqs. (2.7), i.e. $w\left(d_{R}, t\right)-w_{g}(\zeta, t)=0$ and $w\left(d_{F}, t\right)-w_{g}(\zeta+d, t)=0$, extra terms are added to the functional $\mathcal{L}$ using the Lagrange multipliers. Accordingly, the Lagrangian functional $\mathcal{L}$ with multipliers $\lambda_{1}$ and $\lambda_{2}$ is further expressed as

$$
\begin{aligned}
\mathcal{L}= & \left(K_{v}+K_{g}\right)-\left(V_{v}+V_{g}\right)+\lambda_{1} G_{1}+\lambda_{2} G_{2} \\
= & \frac{1}{2} \int_{0}^{L_{v}} \rho_{v} A_{v}\left\{\sum_{j=1}^{N}\left[\boldsymbol{\phi}_{j} \dot{Y}_{j}^{v}+\dot{\zeta} \boldsymbol{\phi}_{j}^{\prime} Y_{j}^{v}\right]\right\}^{2} d x_{1}+\frac{1}{2} m_{v}\left(\dot{\bar{x}}_{r}{ }^{2}+\dot{\bar{y}}_{r}^{2}\right)+\frac{1}{2} J \dot{\bar{\theta}}_{r}^{2} \\
& +\frac{1}{2} \int_{0}^{L_{g}} \rho_{g} A_{g}\left[\sum_{j=1}^{N} \boldsymbol{\psi}_{j} \dot{Y}_{j}^{g}\right]^{2} d x_{2}-\frac{1}{2} \int_{0}^{L_{g}} E_{g} I_{g}\left[\sum_{j=1}^{N} \boldsymbol{\psi}_{j}^{\prime \prime} Y_{j}^{g}\right]^{2} d x_{2} \\
& -\frac{1}{2} \int_{0}^{L_{v}} \sum_{j=1}^{N}\left\{E_{v} I_{v}\left[\boldsymbol{\phi}_{j}^{\prime \prime} Y_{j}^{v}\right]^{2}-P\left[\boldsymbol{\phi}_{j}^{\prime} Y_{j}^{v}\right]^{2}\right\} d x_{1} \\
& -\int_{0}^{L_{v}} \rho_{v} A_{v} g \cos \theta_{E} \sum_{j=1}^{N} \boldsymbol{\phi}_{j} Y_{j}^{v} d x_{1}-\left(m_{v} g \sin \theta_{E}\right) \bar{x}_{r}-\left(m_{v} g \cos \theta_{E}\right) \bar{y}_{r} \\
& +\lambda_{1}\left[\sum_{j=1}^{N} \boldsymbol{\phi}_{j}\left(d_{R}\right) Y_{j}^{v}(t)+y\left(d_{R}, t\right)-\sum_{j=1}^{N} \boldsymbol{\psi}_{j}(\zeta) Y_{j}^{g}(t)\right] \\
& +\lambda_{2}\left[\sum_{j=1}^{N} \boldsymbol{\phi}_{j}\left(d_{F}\right) Y_{j}^{v}(t)+y\left(d_{F}, t\right)-\sum_{j=1}^{N} \boldsymbol{\psi}_{j}(\zeta+d) Y_{j}^{g}(t)\right]
\end{aligned}
$$

where

$$
\begin{gathered}
\bar{x}_{r}(t)=\zeta(t)+r_{1} d \cos \bar{\theta}_{r}(t), \\
y\left(d_{R}, t\right)=\bar{y}_{r}(t)-r_{1} d \sin \bar{\theta}_{r}(t), \\
y\left(d_{F}, t\right)=\bar{y}_{r}(t)+r_{2} d \sin \bar{\theta}_{r}(t), \\
G_{1}=w\left(d_{R}, t\right)-w_{g}(\zeta, t) \\
=\sum_{j=1}^{N} \phi_{j}\left(d_{R}\right) Y_{j}^{v}(t)-\sum_{j=1}^{N} \psi_{j}(\zeta) Y_{j}^{g}(t)+\bar{y}_{r}(t)-r_{1} d \sin \bar{\theta}_{r}(t), \\
G_{2}=w\left(d_{F}, t\right)-w_{g}(\zeta+d, t) \\
=\sum_{j=1}^{N} \phi_{j}\left(d_{F}\right) Y_{j}^{v}(t)-\sum_{j=1}^{N} \psi_{j}(\zeta+d) Y_{j}^{g}(t)+\bar{y}_{r}(t)+r_{2} d \sin \bar{\theta}_{r}(t),
\end{gathered}
$$

$r_{1}=\left(d_{G}-d_{R}\right) / d ; r_{2}=\left(d_{F}-d_{G}\right) / d ; y\left(d_{R}, t\right)$ and $y\left(d_{F}, t\right)$ denote the transverse displacements of the vehicle's rigid body motion at the rear and front shoes, respectively, and $G_{1}$ and $G_{2}$ are the displacement constraints. The two unknowns $\lambda_{1}$ and $\lambda_{2}$ 
can be obtained if Lagrange's equations are solved with the constrained equations. Notably, no damping is considered in the preceding formulation.

Substituting Eqs. (2.7), (2.8), (2.9) and (2.11) into Eq. (2.13), and then substituting the resulting expression for $\mathcal{L}$ into Eq. (2.12) yield

$$
\begin{aligned}
& m_{v} \ddot{\bar{x}}_{r}(t)+m_{v} g \sin \theta_{E}-P(t)=0, \\
& m_{v} \ddot{\bar{y}}_{r}(t)+m_{v} g \cos \theta_{E}-\lambda_{1}-\lambda_{2}=0, \\
& J \ddot{\bar{\theta}}_{r}(t)+\left(r_{1} \lambda_{1}-r_{2} \lambda_{2}\right) d \cos \bar{\theta}_{r}(t)-m_{v} g r_{1} d \sin \theta_{E} \sin \bar{\theta}_{r}(t)=0, \\
& \tilde{H}_{i}^{a} \ddot{\mathrm{Y}}_{i}^{v}(t)+2 \dot{\zeta} \tilde{H}_{i}^{b} \dot{Y}_{i}^{v}(t)+\left(\omega_{v i}^{2} \tilde{H}_{i}^{a}+L_{v} \ddot{\zeta}^{c} \tilde{H}_{i}^{c}+\dot{\zeta}^{2} \tilde{H}_{i}^{c}+\ddot{\zeta} \tilde{H}_{i}^{b}\right) Y_{i}^{v}(t) \\
& \quad-\frac{1}{\rho_{v} A_{v}}\left[\lambda_{1} \phi_{i}\left(d_{R}\right)+\lambda_{2} \phi_{i}\left(d_{F}\right)\right]+g \cos \theta_{E} \tilde{H}_{i}^{d}=0, \\
& \ddot{Y}_{i}^{g}(t)+\omega_{g i}^{2} Y_{i}^{g}(t)+\frac{2}{m_{g}}\left[\lambda_{1} \psi_{i}(\zeta)+\lambda_{2} \psi_{i}(\zeta+d)\right]=0, \\
& \sum_{j=1}^{N} \phi_{j}\left(d_{R}\right) Y_{j}^{v}(t)-\sum_{j=1}^{N} \psi_{j}(\zeta) Y_{j}^{g}(t)+\bar{y}_{r}(t)-r_{1} d \sin \bar{\theta}_{r}(t)=0, \\
& \sum_{j=1}^{N} \phi_{j}\left(d_{F}\right) Y_{j}^{v}(t)-\sum_{j=1}^{N} \psi_{j}(\zeta+d) Y_{j}^{g}(t)+\bar{y}_{r}(t)+r_{2} d \sin \bar{\theta}_{r}(t)=0,
\end{aligned}
$$

where $i=1,2, \ldots, N, m_{g}=\rho_{g} A_{g} L_{g}, \omega_{g i}$ is the circular frequency of the $i$ th mode of the guideway, $\beta_{g i}^{4}=\omega_{g i}^{2} \cdot \frac{\rho_{g} A_{g}}{E_{g} I_{g}}, \beta_{g i} L_{g}=i \pi$, and

$$
\begin{aligned}
\tilde{H}_{i}^{a}= & \frac{1}{2 \beta_{v i}}\left[\left(\Upsilon_{i}^{2}+1\right) \cosh \left(\beta_{v i} L_{v}\right) \sinh \left(\beta_{v i} L_{v}\right)-2 \Upsilon_{i} \cosh ^{2}\left(\beta_{v i} L_{v}\right)\right. \\
& +2\left(\Upsilon_{i}^{2}+1\right) \sin \left(\beta_{v i} L_{v}\right) \cosh \left(\beta_{v i} L_{v}\right)+2 \beta_{v i} L_{v} \\
& \left.-4 \Upsilon_{i} \sin \left(\beta_{v i} L_{v}\right) \sinh \left(\beta_{v i} L_{v}\right)\right], \\
\tilde{H}_{i}^{b}= & \frac{1}{2}\left(\Upsilon_{i}^{2}+1\right) \cosh ^{2}\left(\beta_{v i} L_{v}\right)-\Upsilon_{i} \cosh \left(\beta_{v i} L_{v}\right) \sin \left(\beta_{v i} L_{v}\right)-2 \\
& -\Upsilon_{i} \cosh \left(\beta_{v i} L_{v}\right) \sinh \left(\beta_{v i} L_{v}\right)+\Upsilon_{i}^{2} \sin \left(\beta_{v i} L_{v}\right) \sinh \left(\beta_{v i} L_{v}\right), \\
\tilde{H}_{i}^{c}= & \frac{1}{2} \beta_{v i}\left[\left(\Upsilon_{i}^{2}+1\right) \cosh \left(\beta_{v i} L_{v}\right) \sinh \left(\beta_{v i} L_{v}\right)-2 \Upsilon_{i} \cosh { }^{2}\left(\beta_{v i} L_{v}\right)\right. \\
& \left.+4 \Upsilon_{i}-2 \beta_{v i} L_{v} \Upsilon_{i}^{2}\right], \\
& \tilde{H}_{i}^{d}=\frac{1}{\beta_{v i}}\left[\sin \left(\beta_{v i} L_{v}\right)+\sinh \left(\beta_{v i} L_{v}\right)-\Upsilon_{i} \cosh \left(\beta_{v i} L_{v}\right)\right] .
\end{aligned}
$$

To include the effect of damping in the system of the vehicle and the guideway, an approach that is commonly used in structural dynamics (Clough and Penzien ${ }^{22}$ ) is adopted to add the distributed viscous damping term to Eqs. (2.15d) and (2.15e). 
These equations are thus modified as:

$$
\begin{gathered}
\tilde{H}_{i}^{a} \ddot{\mathrm{Y}}_{i}^{v}(t)+\left(2 \dot{\zeta} \tilde{H}_{i}^{b}+2 \xi_{v} \omega_{v i} \tilde{H}_{i}^{a}\right) \dot{Y}_{i}^{v}(t) \\
+\left(\omega_{v i}^{2} \tilde{H}_{i}^{a}+L_{v} \ddot{\zeta} \tilde{H}_{i}^{c}+\dot{\zeta}^{2} \tilde{H}_{i}^{c}+\ddot{\zeta} \tilde{H}_{i}^{b}\right) Y_{i}^{v}(t) \\
-\frac{1}{\rho_{v} A_{v}}\left[\lambda_{1} \phi_{i}\left(d_{R}\right)+\lambda_{2} \phi_{i}\left(d_{F}\right)\right]+g \cos \theta_{E} \tilde{H}_{i}^{d}=0, \\
\ddot{\mathrm{Y}}_{i}^{g}(t)+2 \xi_{g} \omega_{g i} \dot{Y}_{i}^{g}(t)+\omega_{g i}^{2} Y_{i}^{g}(t)+\frac{2}{m_{g}}\left[\lambda_{1} \psi_{i}(\zeta)+\lambda_{2} \psi_{i}(\zeta+d)\right]=0,
\end{gathered}
$$

where $2 \xi_{v} \omega_{v i}$ and $2 \xi_{g} \omega_{g i}$ are the added damping terms, and $\xi_{v}$ and $\xi_{g}$ are the damping ratios corresponding to the mode shapes $\phi_{i}\left(x_{1}\right)$ and $\psi_{i}\left(x_{2}\right)$, respectively.

Equations (2.15a)-(2.15c), (2.15f), (2.15g) and (2.17) form a set of nonlinear ordinary differential equations for describing the rigid body motions of the vehicle and elastic deformations of the vehicle and the guideway. Equation (2.15a) describes the rigid body motion of the vehicle in the $x_{1}$ direction (see Fig. 2). This equation can be easily derived from Newton's second law based on the free body diagram of the vehicle in Fig. 2, which was utilized to find the solution for $\zeta(t)$ in Eqs. (2.1) - (2.3), where $\zeta(t)=\bar{x}_{r}(t)-r_{1} d$. Consequently, Eqs. (2.15b), (2.15c), (2.15f), (2.15g) and (2.17) can be employed to find the transverse displacements of the vehicle and the guideway. A total of $2 N+4$ equations with $2 N+4$ to-be-determined functions, $Y_{i}^{v}(t)$, $Y_{i}^{g}(t), \bar{y}_{r}(t), \bar{\theta}_{r}(t), \lambda_{1}(t)$ and $\lambda_{2}(t)$, are thus obtained. To solve these equations efficiently, Eqs. (2.15f) and (2.15g) are differentiated twice with respect to time, along with the use of Eqs. (2.17), to yield

$$
\begin{aligned}
\lambda_{1}\left\{\sum_{j=1}^{N}\right. & {\left.\left[d_{2 j} \psi_{j}(\zeta)-d_{1 j} \phi_{j}\left(d_{R}\right)\right]+\frac{1}{m_{v}}+\frac{r_{1}^{2} d^{2} \cos ^{2} \bar{\theta}_{r}(t)}{J}\right\} } \\
& +\lambda_{2}\left\{\sum_{j=1}^{N}\left[e_{2 j} \psi_{j}(\zeta)-e_{1 j} \phi_{j}\left(d_{R}\right)\right]+\frac{1}{m_{v}}-\frac{r_{1} r_{2} d^{2} \cos ^{2} \bar{\theta}_{r}(t)}{J}\right\} \\
& -\sum_{j=1}^{N}\left\{\phi_{j}\left(d_{R}\right)\left[b_{1 j} \dot{Y}_{j}^{v}(t)+c_{1 j} Y_{j}^{v}(t)+f_{1 j}\right]\right. \\
& -\psi_{j}(\zeta)\left[b_{2 j} \dot{Y}_{j}^{g}(t)+c_{2 j} Y_{j}^{g}(t)\right]+2 \dot{\zeta} \psi_{j}^{\prime}(\zeta) \dot{Y}_{j}^{g}(t) \\
& \left.+\left[\ddot{\zeta}_{j}^{\prime}(\zeta)+\dot{\zeta}^{2} \psi_{j}^{\prime \prime}(\zeta)\right] Y_{j}^{g}(t)\right\}-\frac{m_{v} g r_{1}^{2} d^{2} \sin \theta_{E} \sin \bar{\theta}_{r}(t) \cos \bar{\theta}_{r}(t)}{J} \\
& +r_{1} d \dot{\bar{\theta}}_{r}^{2}(t) \sin _{r}(t)-g \cos \theta_{E}=0, \\
& \lambda_{1}\left\{\sum_{j=1}^{N}\left[d_{2 j} \psi_{j}(\zeta+d)-d_{1 j} \phi_{j}\left(d_{F}\right)\right]+\frac{1}{m_{v}}-\frac{r_{1} r_{2} d^{2} \cos ^{2} \bar{\theta}_{r}(t)}{J}\right\} \\
& +\lambda_{2}\left\{\sum_{j=1}^{N}\left[e_{2 j} \psi_{j}(\zeta+d)-e_{1 j} \phi_{j}\left(d_{F}\right)\right]+\frac{1}{m_{v}}+\frac{r_{2}^{2} d^{2} \cos ^{2} \bar{\theta}_{r}(t)}{J}\right\}
\end{aligned}
$$




$$
\begin{aligned}
& -\sum_{j=1}^{N}\left\{\phi_{j}\left(d_{F}\right)\left[b_{1 j} \dot{Y}_{j}^{v}(t)+c_{1 j} Y_{j}^{v}(t)+f_{1 j}\right]\right. \\
& -\psi_{j}(\zeta+d)\left[b_{2 j} \dot{Y}_{j}^{g}(t)+c_{2 j} Y_{j}^{g}(t)\right]+2 \dot{\zeta} \psi_{j}^{\prime}(\zeta+d) \dot{Y}_{j}^{g}(t) \\
& \left.+\left[\ddot{\zeta} \psi_{j}^{\prime}(\zeta+d)+\dot{\zeta}^{2} \psi_{j}^{\prime \prime}(\zeta+d)\right] Y_{j}^{g}(t)\right\} \\
& +\frac{m_{v} g r_{1} r_{2} d^{2} \sin \theta_{E} \sin \bar{\theta}_{r}(t) \cos \bar{\theta}_{r}(t)}{J} \\
& -r_{2} d \dot{\bar{\theta}}_{r}^{2}(t) \sin \bar{\theta}_{r}(t)-g \cos \theta_{E}=0,
\end{aligned}
$$

where

$$
\begin{aligned}
& b_{1 j}=2 \xi_{v} \omega_{v j}+\frac{2 \dot{\zeta} \tilde{H}_{j}^{b}}{\tilde{H}_{j}^{a}}, \quad c_{1 j}=\omega_{v j}^{2}+\frac{L_{v} \ddot{\zeta} \tilde{H}_{j}^{c}+\dot{\zeta}^{2} \tilde{H}_{j}^{c}+\ddot{\zeta}_{j}^{b}}{\tilde{H}_{j}^{a}}, \\
& d_{1 j}=-\frac{\phi_{j}\left(d_{R}\right)}{\rho_{v} A_{v} \tilde{H}_{j}^{a}}, \quad e_{1 j}=-\frac{\phi_{j}\left(d_{F}\right)}{\rho_{v} A_{v} \tilde{H}_{j}^{a}}, \quad f_{1 j}=\frac{g \cos \theta_{E} \tilde{H}_{j}^{d}}{\tilde{H}_{j}^{a}}, \\
& b_{2 j}=2 \xi_{g} \omega_{g j}, \quad c_{2 j}=\omega_{g j}^{2}, \quad d_{2 j}=\frac{2 \psi_{j}(\zeta)}{m_{g}}, \quad e_{2 j}=\frac{2 \psi_{j}(\zeta+d)}{m_{g}} .
\end{aligned}
$$

Consequently, Eqs. (2.15b), (15c), (2.17) and (2.18) are used to determine the transverse motions of the vehicle and the guideway for $0<t \leq t_{F}$, as discussed below.

To solve the above governing equations, the initial conditions are required. The system (vehicle and guideway) is initially at rest, i.e. the initial velocity and acceleration of the vehicle and the guideway are zero. However, the vehicle and guideway are both deformed under the weight of the vehicle. The initial displacement of the system can be determined from Eqs. (2.15b), (15c), (2.15f), (2.15g) and (2.17) by setting $\ddot{\mathrm{Y}}_{i}^{g}(0)=0, \dot{Y}_{i}^{g}(0)=0, \ddot{\mathrm{Y}}_{i}^{v}(0)=0, \dot{Y}_{i}^{v}(0)=0, \ddot{\zeta}(0)=0$ and $\dot{\zeta}(0)=0$. Then, the initial values of $Y_{i}^{g}(0), Y_{i}^{v}(0), \bar{y}_{r}(0), \bar{\theta}_{r}(0), \lambda_{1}(0)$ and $\lambda_{2}(0)$ can be easily determined.

\subsection{Tip-off phase}

Figure 4 shows the free body diagram of the vehicle and the guideway for $t_{F}<t \leq t_{R}$, when the front shoe of the vehicle has lost contact with the guideway, while the rear shoe remains in contact with the guideway. Similar to that in the twoshoe contact phase, the Lagrangian functional $\mathcal{L}$ in the tip-off phase is

$$
\begin{aligned}
\mathcal{L}= & \left(K_{v}^{*}+K_{g}^{*}\right)-\left(V_{v}^{*}+V_{g}^{*}\right)+\lambda_{1}^{*} G_{1}^{*} \\
= & \frac{1}{2} \int_{0}^{L_{v}} \rho_{v} A_{v}\left\{\sum_{j=1}^{N}\left[\phi_{j} \dot{Y}_{j}^{v *}+\dot{\zeta} \phi_{j}^{\prime} Y_{j}^{v *}\right]\right\}^{2} d x_{1}+\frac{1}{2} m_{v}\left(\dot{\bar{x}}_{r}^{* 2}+\dot{\bar{y}}_{r}^{*} 2\right)+\frac{1}{2} J \dot{\bar{\theta}}_{r}^{* 2} \\
& +\frac{1}{2} \int_{0}^{L_{g}} \rho_{g} A_{g}\left[\sum_{j=1}^{N} \psi_{j} \dot{Y}_{j}^{g *}\right]^{2} d x_{2}-\frac{1}{2} \int_{0}^{L_{g}} E_{g} I_{g}\left[\sum_{j=1}^{N} \psi_{j}^{\prime \prime} Y_{j}^{g *}\right]^{2} d x_{2}
\end{aligned}
$$




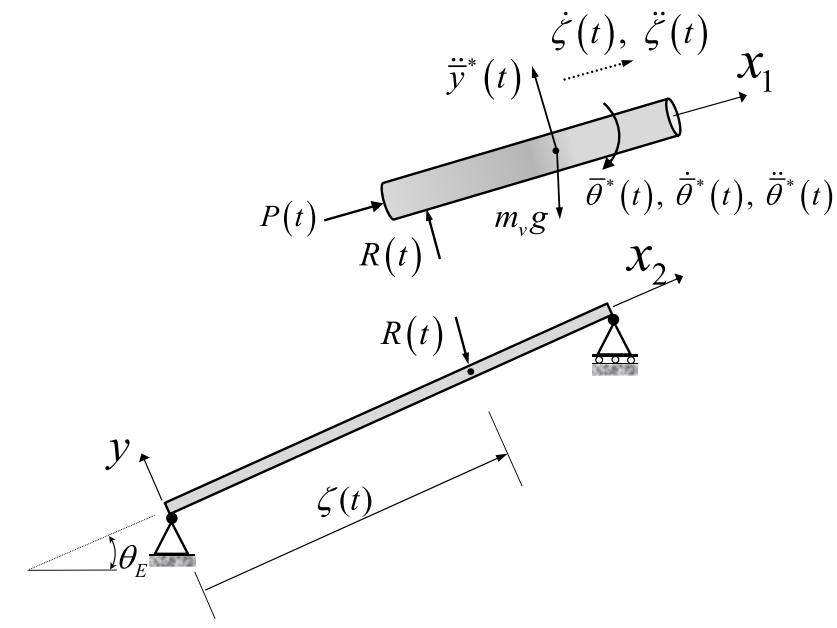

Fig. 4. Free body diagrams of the system in tip-off phase.

$$
\begin{aligned}
& -\frac{1}{2} \int_{0}^{L_{v}} \sum_{j=1}^{N}\left\{E_{v} I_{v}\left[\phi_{j}^{\prime \prime} Y_{j}^{v *}\right]^{2}-P\left[\phi_{j}^{\prime} Y_{j}^{v *}\right]^{2}\right\} d x_{1} \\
& -\int_{0}^{L_{v}} \rho_{v} A_{v} g \cos \theta_{E} \sum_{j=1}^{N} \phi_{j} Y_{j}^{v *} d x_{1}-\left(m_{v} g \sin \theta_{E}\right) \bar{x}_{r}^{*}-\left(m_{v} g \cos \theta_{E}\right) \bar{y}_{r}^{*} \\
& +\lambda_{1}^{*}\left[\sum_{j=1}^{N} \phi_{j}\left(d_{R}\right) Y_{j}^{v *}(t)-\sum_{j=1}^{N} \psi_{j}(\zeta) Y_{j}^{g *}(t)+\bar{y}_{r}^{*}(t)-r_{1} d \sin \bar{\theta}_{r}^{*}(t)\right]
\end{aligned}
$$

where the superscript $*$ stands for quantities in the tip-off phase. Since the front shoe of the vehicle has lost contact with the guideway, the constraint on displacement, given in Eq. (2.7b), vanishes. Following the procedure described in the preceding section, the following governing equations are obtained:

$$
\begin{gathered}
m_{v} \ddot{\bar{x}}_{r}^{*}(t)+m_{v} g \sin \theta_{E}-P(t)=0, \\
m_{v} \ddot{\bar{y}}_{r}^{*}(t)+m_{v} g \cos \theta_{E}-\lambda_{1}^{*}=0, \\
J \ddot{\bar{\theta}}_{r}^{*}(t)+\lambda_{1}^{*} r_{1} d \cos \bar{\theta}_{r}^{*}(t)-m_{v} g r_{1} d \sin \theta_{E} \sin \bar{\theta}_{r}^{*}(t)=0, \\
\tilde{H}_{i}^{a} \ddot{\mathrm{Y}}_{i}^{v *}(t)+\left(2 \xi_{v} \omega_{v i} \tilde{H}_{i}^{a}+2 \dot{\zeta} \tilde{H}_{i}^{b}\right) \dot{Y}_{i}^{v *}(t)+\left(\omega_{v i}^{2} \tilde{H}_{i}^{a}+L_{v} \ddot{\zeta} \tilde{H}_{i}^{c}+\dot{\zeta}^{2} \tilde{H}_{i}^{c}\right. \\
\left.+\ddot{\zeta} \tilde{H}_{i}^{b}\right) Y_{i}^{v *}(t)-\frac{\lambda_{1}^{*} \phi_{i}\left(d_{R}\right)}{\rho_{v} A_{v}}+g \cos \theta_{E} \tilde{H}_{i}^{d}=0, \\
\ddot{\mathrm{Y}}_{i}^{g *}(t)+2 \xi_{g} \omega_{g i} \dot{Y}_{i}^{g *}(t)+\omega_{g i}^{2} Y_{i}^{g *}(t)+\frac{2 \lambda_{1}^{*} \psi_{i}(\zeta)}{m_{g}}=0, \\
\sum_{j=1}^{N} \phi_{j}\left(d_{R}\right) Y_{j}^{v *}(t)-\sum_{j=1}^{N} \psi_{j}(\zeta) Y_{j}^{g *}(t)+\bar{y}_{r}^{*}(t)-r_{1} d \sin \bar{\theta}_{r}^{*}(t)=0 .
\end{gathered}
$$


Again, Eq. (2.21a) is not needed for determining the transverse displacements of the vehicle and the guideway. Differentiating Eq. (2.21f) twice with respect to time and applying Eqs. (2.21d) and (2.21e) yield

$$
\begin{aligned}
\lambda_{1}^{*}\left\{\sum_{j=1}^{N}\left[d_{2 j}^{*} \psi_{j}(\zeta)-d_{1 j}^{*} \phi_{j}\left(d_{R}\right)\right]+\frac{1}{m_{v}}+\frac{r_{1}^{2} d^{2} \cos ^{2} \bar{\theta}_{r}^{*}(t)}{J}\right\} \\
\quad-\sum_{j=1}^{N}\left\{\phi_{j}\left(d_{R}\right)\left[b_{1 j}^{*} \dot{Y}_{j}^{v *}(t)+c_{1 j}^{*} Y_{j}^{v *}(t)+f_{1 j}^{*}\right]\right. \\
\quad-\psi_{j}(\zeta)\left[b_{2 j}^{*} \dot{Y}_{j}^{g *}(t)+c_{2 j}^{*} Y_{j}^{g *}(t)\right]+2 \dot{\zeta} \psi_{j}^{\prime}(\zeta) \dot{Y}_{j}^{g *}(t) \\
\left.\quad+\left[\ddot{\zeta} \psi_{j}^{\prime}(\zeta)+\dot{\zeta}^{2} \psi_{j}^{\prime \prime}(\zeta)\right] Y_{j}^{g *}(t)\right\}-\frac{m_{v} g r_{1}^{2} d^{2} \sin \theta_{E} \sin \bar{\theta}_{r}^{*}(t) \cos \bar{\theta}_{r}^{*}(t)}{J} \\
\quad+r_{1} d \dot{\bar{\theta}}_{r}^{* 2}(t) \sin \bar{\theta}_{r}^{*}(t)-g \cos \theta_{E}=0,
\end{aligned}
$$

where $d_{1 j}^{*}=d_{1 j}, e_{1 j}^{*}=e_{1 j}, f_{1 j}^{*}=f_{1 j}, b_{2 j}^{*}=b_{2 j}, c_{2 j}^{*}=c_{2 j}$, and

$$
\begin{gathered}
b_{1 j}^{*}=2 \xi_{v} \omega_{v j}+\frac{2 \dot{\zeta} \tilde{H}_{j}^{b}}{\tilde{H}_{j}^{a}}, \quad c_{1 j}^{*}=\omega_{v j}^{2}+\frac{L_{v} \ddot{\zeta} \tilde{H}_{j}^{c}+\dot{\zeta}^{2} \tilde{H}_{j}^{c}+\ddot{\zeta} \tilde{H}_{j}^{b}}{\tilde{H}_{j}^{a}}, \\
d_{2 j}^{*}=\frac{2 \psi_{j}(\zeta)}{m_{g}}, \quad e_{2 j}^{*}=\frac{2 \psi_{j}(\zeta+d)}{m_{g}} .
\end{gathered}
$$

Equations (2.21b)-(2.21e) and Eq. (2.22) describe the transverse motions of the vehicle and the guideway in the tip-off phase; they form a set of $2 N+3$ nonlinear equations. The initial conditions for solving these equations are obtained from the continuity conditions between the motions in the two phases: $Y_{j}^{v *}(0)=Y_{j}^{v}\left(t_{F}^{+}\right)$, $Y_{j}^{g *}(0)=Y_{j}^{g}\left(t_{F}^{+}\right), \quad \bar{y}_{r}^{*}(0)=\bar{y}_{r}\left(t_{F}^{+}\right), \quad \bar{\theta}_{r}^{*}(0)=\bar{\theta}_{r}\left(t_{F}^{+}\right), \quad \dot{Y}_{j}^{v *}(0)=\dot{Y}_{j}^{v}\left(t_{F}^{+}\right), \quad \dot{Y}_{j}^{g *}(0)=$ $\dot{Y}_{j}^{g}\left(t_{F}^{+}\right), \dot{\bar{y}}_{r}^{*}(0)=\dot{\bar{y}}_{r}\left(t_{F}^{+}\right)$and $\dot{\bar{\theta}}_{r}^{*}(0)=\dot{\bar{\theta}}_{r}\left(t_{F}^{+}\right)$.

\subsection{Dynamic responses of vehicle and guideway}

The governing equations given in Secs. 2.2 and 2.3 form a set of coupled second order differential nonlinear equations. They can be expressed in matrix form as

$$
\mathbf{M} \ddot{\mathbf{Y}}+\mathbf{C} \dot{\mathbf{Y}}+\mathbf{K Y}=\mathbf{Q},
$$

where $\mathbf{Y}, \dot{\mathbf{Y}}$ and $\ddot{\mathbf{Y}}$ are the generalized coordinate, velocity and acceleration vectors, respectively; $\mathbf{Q}$ is a generalized force vector, and $\mathbf{M}, \mathbf{C}$ and $\mathbf{K}$ are the instantaneous overall mass, damping and stiffness matrixes, respectively. Equation (2.24) is further reduced to a set of first-order differential equations in matrix form by introducing $\mathbf{Z}=\left(\mathbf{Y}^{T}, \dot{\mathbf{Y}}^{T}\right)^{T}$. Then, the Petzold-Gear BDF method ${ }^{20}$ is applied to solving the resulting equations.

After $\mathbf{Y}$ and $\dot{\mathbf{Y}}$ have been determined, the vertical displacement and velocity of the center of gravity of the vehicle can be determined by applying the following 
equations, respectively;

$$
\begin{gathered}
w\left(d_{G}, t\right)=\bar{y}_{r}(t)+w_{v}\left(d_{G}, t\right)=\bar{y}_{r}(t)+\sum_{j=1}^{N} \phi_{j}\left(d_{G}\right) Y_{j}^{v}(t), \\
\dot{w}\left(d_{G}, t\right)=\dot{\bar{y}}_{r}(t)+\dot{w}_{v}\left(d_{G}, t\right)=\dot{\bar{y}}_{r}(t)+\sum_{j=1}^{N}\left[\phi_{j}\left(d_{G}\right) \dot{Y}_{j}^{v}(t)+\dot{\zeta} \phi_{j}^{\prime}\left(d_{G}\right) Y_{j}^{v}(t)\right] .
\end{gathered}
$$

The pitch angle and pitch rate of the vehicle are found, respectively, by applying

$$
\begin{gathered}
\theta(t)=\bar{\theta}_{r}(t)+\sin ^{-1}\left\{\frac{1}{d} \sum_{j=1}^{N}\left[\phi_{j}\left(d_{F}\right)-\phi_{j}\left(d_{R}\right)\right] Y_{j}^{v}(t)\right\}, \\
\dot{\theta}(t)=\dot{\bar{\theta}}_{r}(t)+\frac{1}{d \cos \left[\theta(t)-\bar{\theta}_{r}(t)\right]} \sum_{j=1}^{N}\left\{\left[\phi_{j}\left(d_{F}\right)-\phi_{j}\left(d_{R}\right)\right] \dot{Y}_{j}^{v}(t)\right. \\
\left.+\dot{\zeta}\left[\phi_{j}^{\prime}\left(d_{F}\right)-\phi_{j}^{\prime}\left(d_{R}\right)\right] Y_{j}^{v}(t)\right\} .
\end{gathered}
$$

\section{Numerical Validation and Examples}

In this section, three cases will be studied to validate the method proposed, by comparing the results with those by Yao and Zhang ${ }^{18}$ and Chou et al. ${ }^{19}$ As mentioned in the introduction section, two typical models were applied to study the tipoff phenomenon of a vehicle when it moves along the guideway. Yao and Zhang utilized the model of a rigid vehicle moving along a rigid guideway (the R.R. model) ${ }^{18}$ while Chou et al. adopted the model of a rigid vehicle moving on an elastic guideway (R.E. model). ${ }^{19}$ In this study, the vehicle and guideway are assumed to be elastic, and are simulated by the finite elements. Unless noted otherwise, the material and geometric parameters of the vehicle and guideway and the parameters defining a typical thrust-time diagram (Fig. 3) are those given in Table 1 . Ten modes $(N=10$ in Eq. (2.8)) and a time increment of $0.0001 \mathrm{~s}$ were used to obtain the results.

\subsection{Case 1: A rigid vehicle moves along a rigid guideway}

In this case, the flexural rigidities of the vehicle and guideway are assumed to be $1.2 \times 10^{15} \mathrm{~N} \cdot \mathrm{m}^{2}$ to simulate the behavior of a pseudo-rigid body. Figures 5 and 6 display the numerical results for the pitch angle and pitch rate, respectively, of the vehicle obtained using three different models. In the two-shoe contact phase, the pitch angle and pitch rate of the vehicle in the R.R. model are theoretically zero, while in the R.E. and E.E. models, they have very small values, because the vehicle and guideway have very large flexural rigidities.

The results by Yao and Zhang ${ }^{18}$ (R.R. model) for the tip-off phase differ significantly from those based on the other models. The former are somewhat inconsistent with the physical consideration in that a rigid vehicle should maintain its uniform 
Table 1. Parameters of the vehicle launch system.

\begin{tabular}{lcc}
\hline & \multicolumn{2}{c}{ Design value of launch system } \\
\cline { 2 - 3 } Parameters & Vehicle & Guideway \\
\hline$E_{*} I_{*}$ & $1.2 \times 10^{7} \mathrm{~N} \cdot \mathrm{m}^{2}$ & $1.2 \times 10^{7} \mathrm{~N} \cdot \mathrm{m}^{2}$ \\
$\rho_{*} A_{*}$ & $4.0 \times 10^{2} \mathrm{~kg} / \mathrm{m}$ & $1.5 \times 10^{2} \mathrm{~kg} / \mathrm{m}$ \\
$\xi_{*}$ & 0.03 & 0.03 \\
$L_{*}$ & $4.0 \mathrm{~m}$ & $8.0 \mathrm{~m}$ \\
$\theta_{E}$ & - & $0.5 \mathrm{rad}$ \\
$d$ & $3.7 \mathrm{~m}$ & - \\
$r_{1} d$ & $2.5 \mathrm{~m}$ & - \\
$\zeta_{R}$ & - & $0.1 \mathrm{~m}$ \\
$\zeta_{F}$ & - & $4.2 \mathrm{~m}$ \\
$t_{b}$ & $0.1 \mathrm{~s}$ & - \\
$P_{\max }$ & $7.0 \times 10^{4} \mathrm{~N}$ & - \\
$\Delta t$ & $0.0001 \mathrm{~s}$ & - \\
\hline
\end{tabular}

*The subscript denotes the vehicle $(v)$ or guideway $(g)$.

rotational acceleration about its rear shoe when the front shoe loses contact with the rigid guideway. Consequently, the slope of the pitch rate in Fig. 6 should be constant. A nearly straight line was revealed by both the present results and those of Chou et al. ${ }^{19}$ whereas those of Yao and Zhang ${ }^{18}$ do not show a straight line.

\subsection{Case 2: A rigid vehicle moves along an elastic guideway}

Figures 7 and 8 show the time histories of the pitch angle and pitch rate of vehicle obtained by the R.E. and E.E. models. For comparison, the flexural rigidity of the vehicle is set equal to $1.2 \times 10^{15} \mathrm{~N} \cdot \mathrm{m}^{2}$ in the E.E. model. The pitch angle and pitch

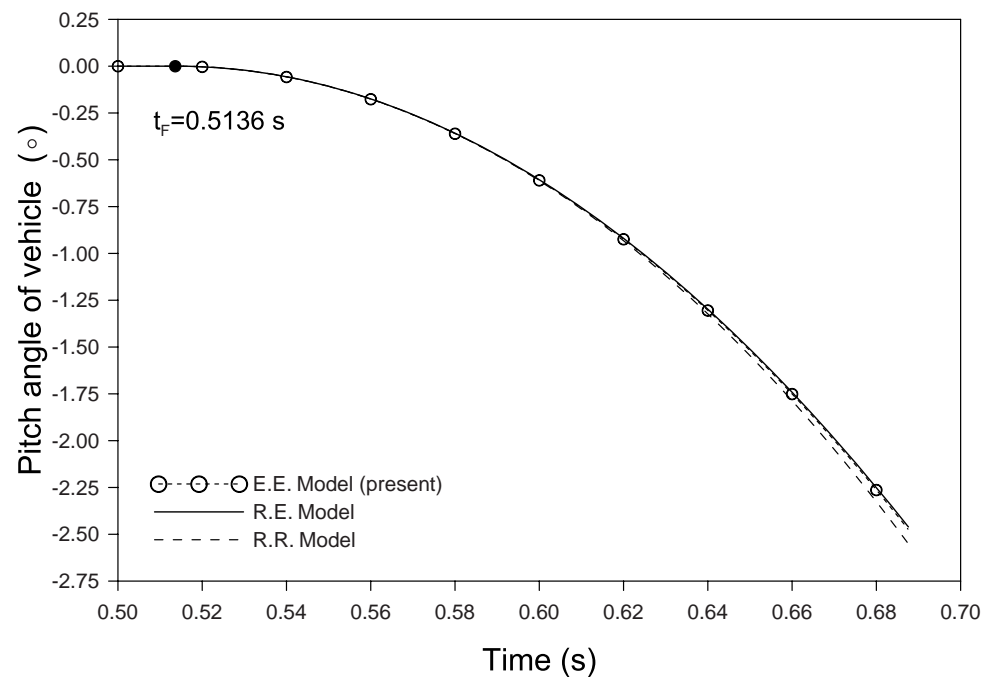

Fig. 5. Pitch angle $\theta-t$ of vehicle obtained using different models. 


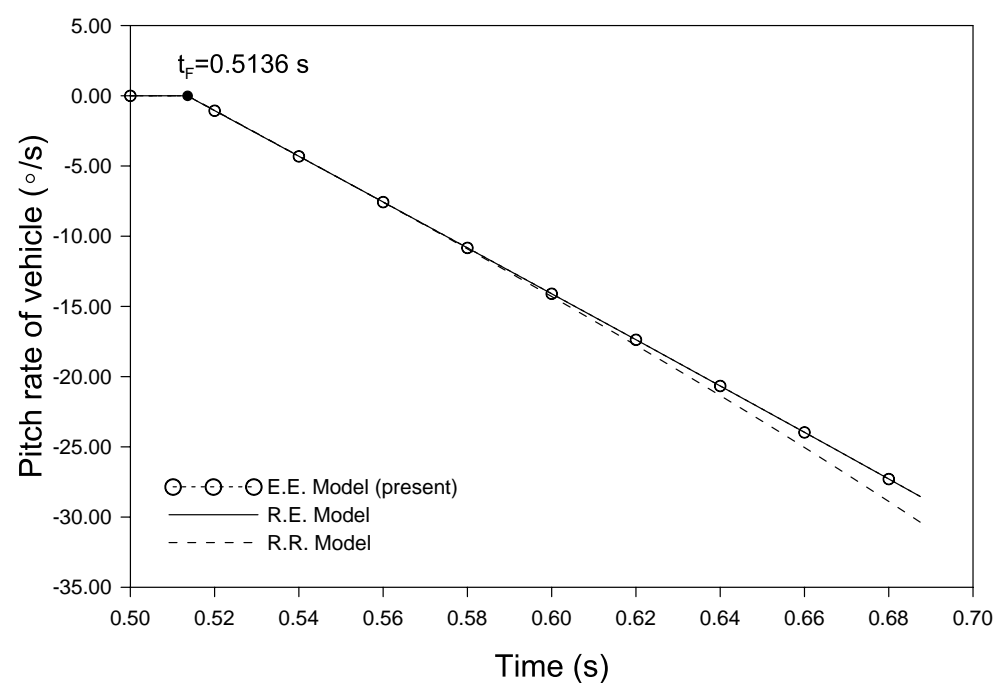

Fig. 6. Pitch rate $\dot{\theta}-t$ of vehicle obtained using different models.

rate of the vehicle are directly obtained from Eqs. (2.27) and (2.28), referred to as the "vehicle formulation". Besides, the pitch angle and pitch rate of the vehicle for the R.E. model were indirectly determined from the displacements of the guideway at the points of contact with the shoes of the vehicle, ${ }^{19}$ which was referred to as the "guideway formulation". For consistency, the pitch angle and pitch rate of the vehicle in the E.E. model were also computed using the "guideway formulation".

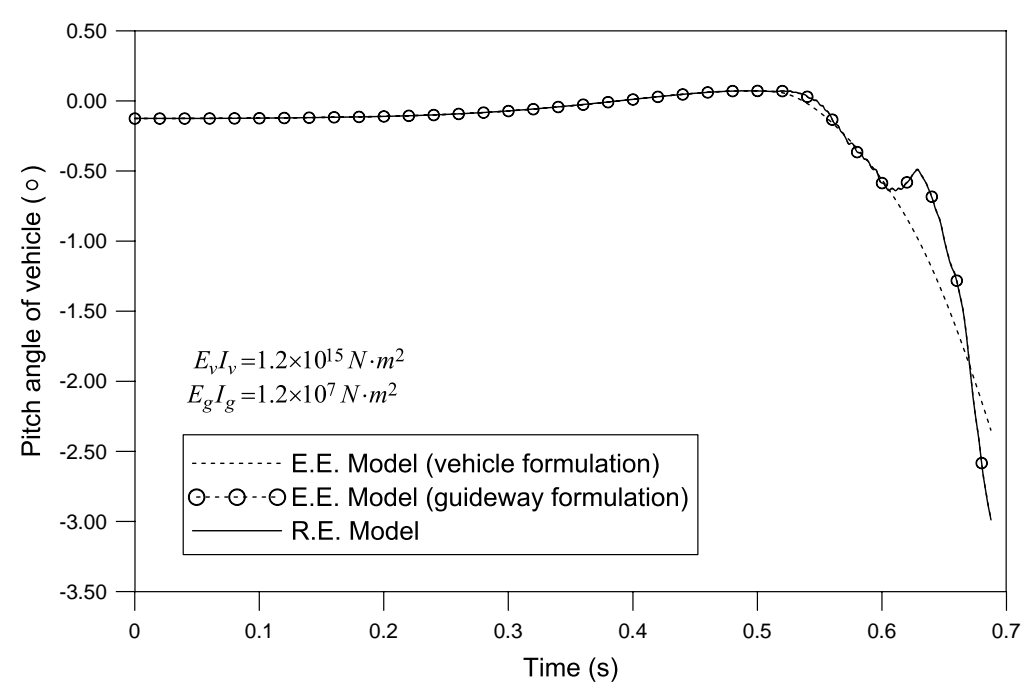

Fig. 7. Pitch angle $\theta-t$ of vehicle obtained using different models and formulations. 


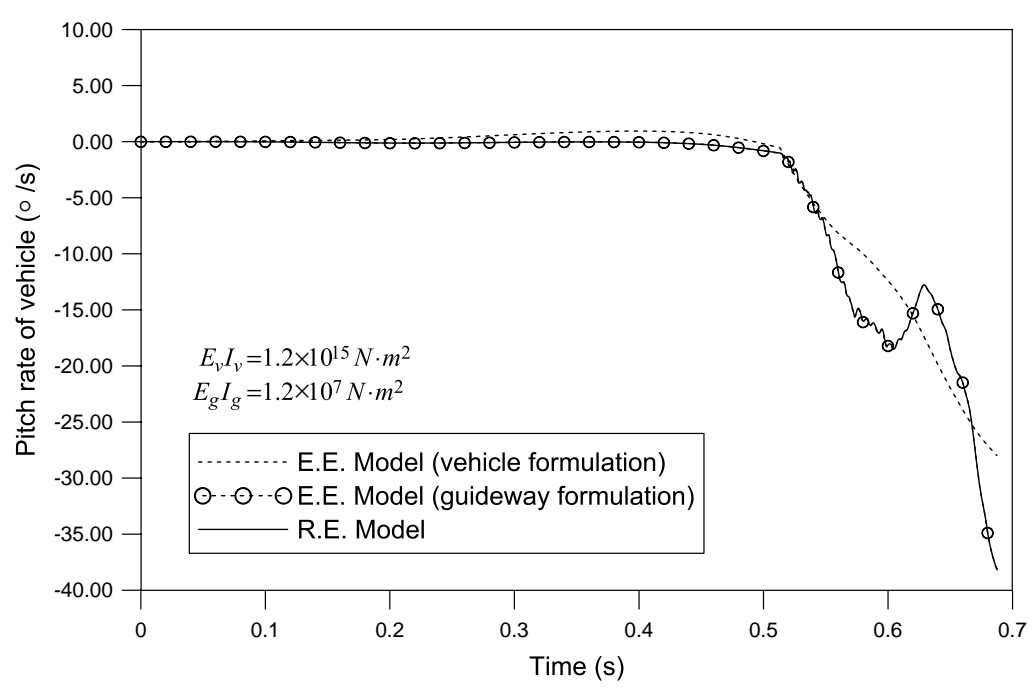

Fig. 8. Pitch rate $\dot{\theta}-t$ of vehicle obtained using different models and formulations.

The excellent agreement between the results of the R.E. model and those of the E.E. model based on the "guideway formulation" confirms the accuracy of the proposed approach. The considerable differences in the tip-off phase between the results of the R.E. model and those of the E.E. model based on the "vehicle formulation" indicate the importance of the present approach in predicting the dynamic response of the vehicle.

\subsection{Case 3: An elastic vehicle moves along an elastic guideway}

Case 3 is concerned with the motion of an elastic vehicle moving along an elastic guideway. Table 1 presents the material properties and geometric parameters of the vehicle and guideway. In Fig. 9, the pitch angles obtained by using a time increment of $0.0001 \mathrm{~s}$ and 10 modes are compared with those using 1 and 50 modes, which indicates that the solution can be obtained accurately using 10 modes. In Fig. 10, the pitch angles obtained by using 10 modes and a time increment of $0.0001 \mathrm{~s}$ are compared with those using the time increments of $\Delta t=0.01,0.001,0.0001$ and $0.00001 \mathrm{~s}$, which indicates that accurate solutions can be obtained using a time increment of $0.0001 \mathrm{~s}$.

In Figs. 11 and 12, the pitch angles and pitch rates of the vehicle obtained using three models - R.R., R.E. and E.E. are compared. The tip-off phase starts at $t_{F}=$ $0.5136 \mathrm{~s}$ and ends at $t_{R}=0.6876 \mathrm{~s}$. In the two-shoe contact phase, the R.R. model only considers the rigid body motions so that the pitch angles and pitch rates of the vehicle equal zero, thereby underestimating the magnitude of the pitch angle of the vehicle, while the E.E. model includes the elastic deformations of the vehicle and guideway and yields a higher result for the pitch rate of the vehicle than the other 


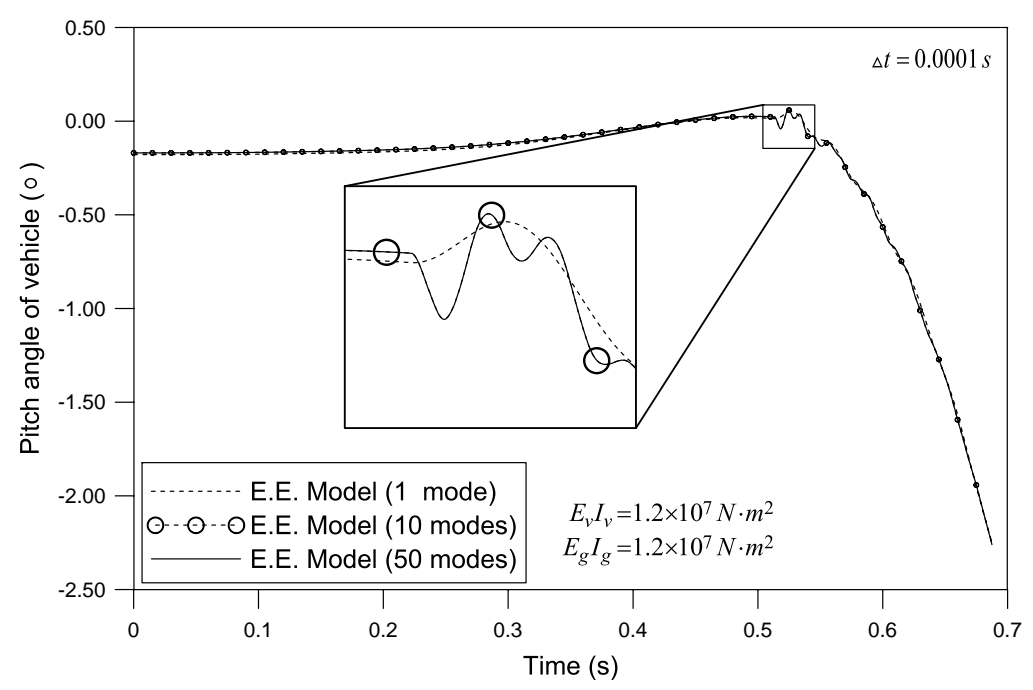

Fig. 9. Pitch angles of vehicle obtained using different numbers of modes.

two models. In the tip-off phase, the agreement between the results obtained by the R.R. and E.E. models is better than that between those obtained by the R.E. and E.E. models. The maximum difference in the pitch angle between the results of the R.E. and E.E. models is $0.5780^{\circ}$ at $t=0.6350 \mathrm{~s}$, while the maximum difference in the pitch rate is $10.2670^{\circ} / \mathrm{s}$ at $t=0.6876 \mathrm{~s}$. The considerable differences between the results obtained by the R.E. and E.E. models are mainly due to the fact that, as shown in Sec. 3.2, different formulations are employed to determine the pitch angles

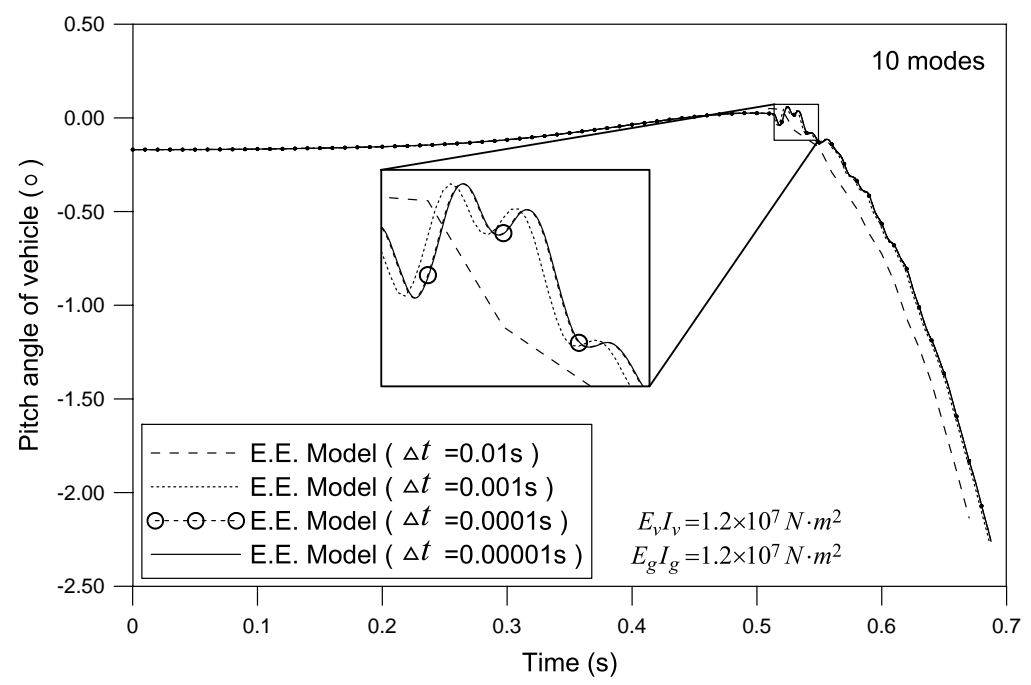

Fig. 10. Pitch angles of vehicle obtained using different time increments. 


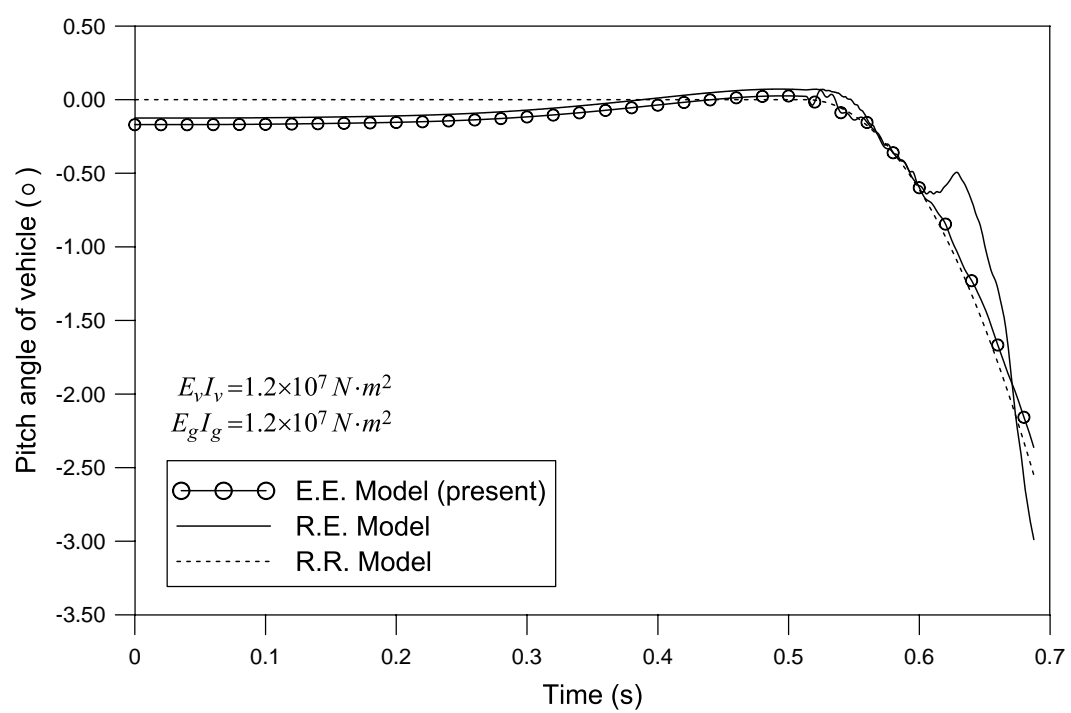

Fig. 11. Pitch angles of vehicle obtained using different models.

and pitch rates of the vehicle. However, at the end of the tip-off phase $(t=0.6876 \mathrm{~s})$, the E.E., R.E. and R.R. models yield the pitch angles as $-2.3622^{\circ},-2.9886^{\circ}$ and $-2.5546^{\circ}$, respectively, and the pitch rates as $-27.914^{\circ} / \mathrm{s},-38.181^{\circ} / \mathrm{s}$ and $-30.410^{\circ} / \mathrm{s}$. These differences significantly influence the trajectory of the vehicle after it leaves the guideway.

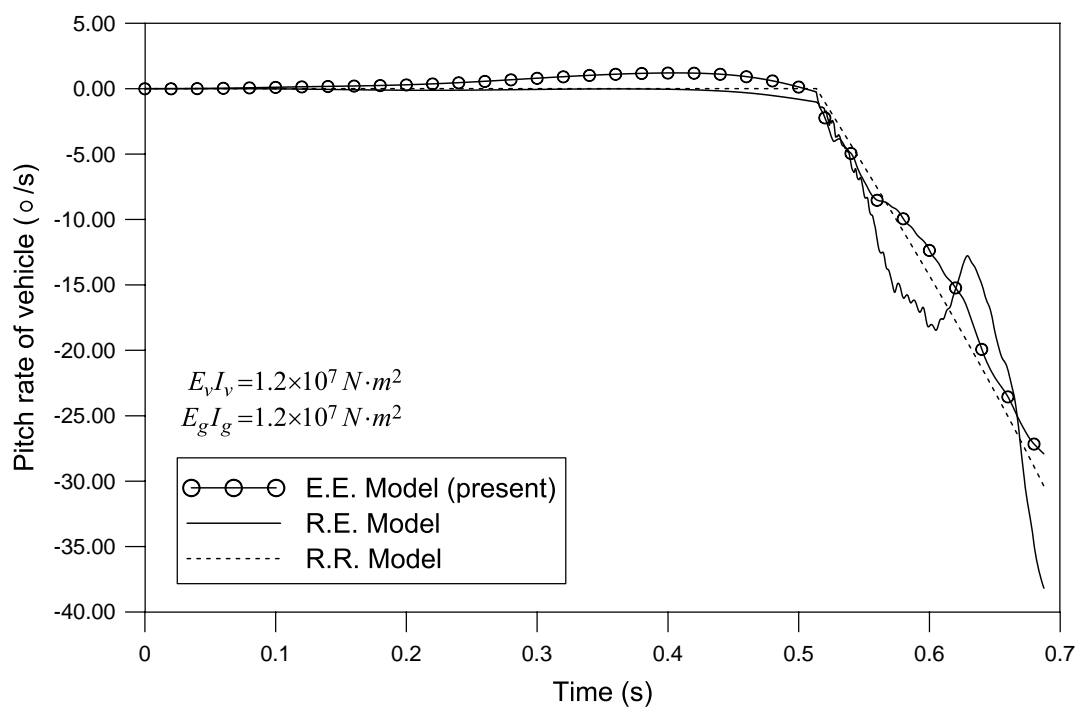

Fig. 12. Pitch rates of vehicle obtained using different models. 


\section{Parametric Study}

After the accuracy of the proposed approach was confirmed, the solutions are utilized to examine the effects of some important parameters on the pitch angle and pitch rate of the vehicle at take-off. The parameters of interest are the length of the guideway, $L_{g}$; the distance between the shoes of the vehicle, $d$, the mass ratio, $M_{r}$, defined as $\rho_{v} A_{v} L_{v} / \rho_{g} A_{g} L_{g}$, and the flexural rigidity ratio, $R_{r}$, defined as $E_{v} I_{v} / E_{g} I_{g}$. In control engineering, the pitch angle and pitch rate of a vehicle at take-off are the main concerns because they are the factors that dominate the trajectory of the vehicle after it leaves the guideway.

\subsection{Influence of length of guideway}

The length of the guideway affects the duration of a vehicle's movement along the guideway. Increasing the length of the guideway increases the period for which the two shoes are in contact with the guideway. Increasing the length of the guideway also increases the velocity of the vehicle when it enters the tip-off phase, because the period for which the motor thrust acts is increased, while the duration $t_{R}-t_{F}$ is reduced. Consequently, the length of the guideway substantially affects the tip-off response of the vehicle.

Table 2 presents three combinations of flexural rigidities of the vehicle and guideway considered herein. Case EI01 is used to represent a flexible vehicle and a flexible guideway; case EI02 a rigid vehicle and a flexible guideway; and case EI03 a rigid vehicle and a rigid guideway.

Figures 13 and 14, respectively, show the variations of the pitch angle and pitch rate of vehicle at take-off for a guideway length between 4 and $12 \mathrm{~m}$. In Tables 1 and 2 , the other parameters that must be known to solve for the dynamic response of the vehicle and the guideway are listed. Both the pitch angle and pitch rate of the vehicle at take-off generally decrease as the guideway length increases, since the duration $t_{R}-t_{F}$ decreases. Accordingly, a longer guideway is associated with a weaker vehicle tip-off effect. Nevertheless, the length of the guideway should still be selected to fit the spatial limits on the launcher system.

The results given in Figs. 13 and 14 also reveal that the flexural rigidity combination EI02 always yields a smaller pitch angle and pitch rate of the vehicle than the combination EI03. Combination EI01 yields results that may be larger or smaller

Table 2. Combinations of flexural rigidities of vehicle and guideway.

\begin{tabular}{lcc}
\hline & \multicolumn{2}{c}{ Flexural rigidity $\left(\mathrm{N} \cdot \mathrm{m}^{2}\right)$} \\
\cline { 2 - 3 } Case & $E_{v} I_{v}$ & $E_{g} I_{g}$ \\
\hline EI01 & $1.2 \times 10^{06}$ & $1.2 \times 10^{07}$ \\
EI02 & $1.2 \times 10^{15}$ & $1.2 \times 10^{06}$ \\
EI03 & $1.2 \times 10^{15}$ & $1.2 \times 10^{15}$ \\
\hline
\end{tabular}




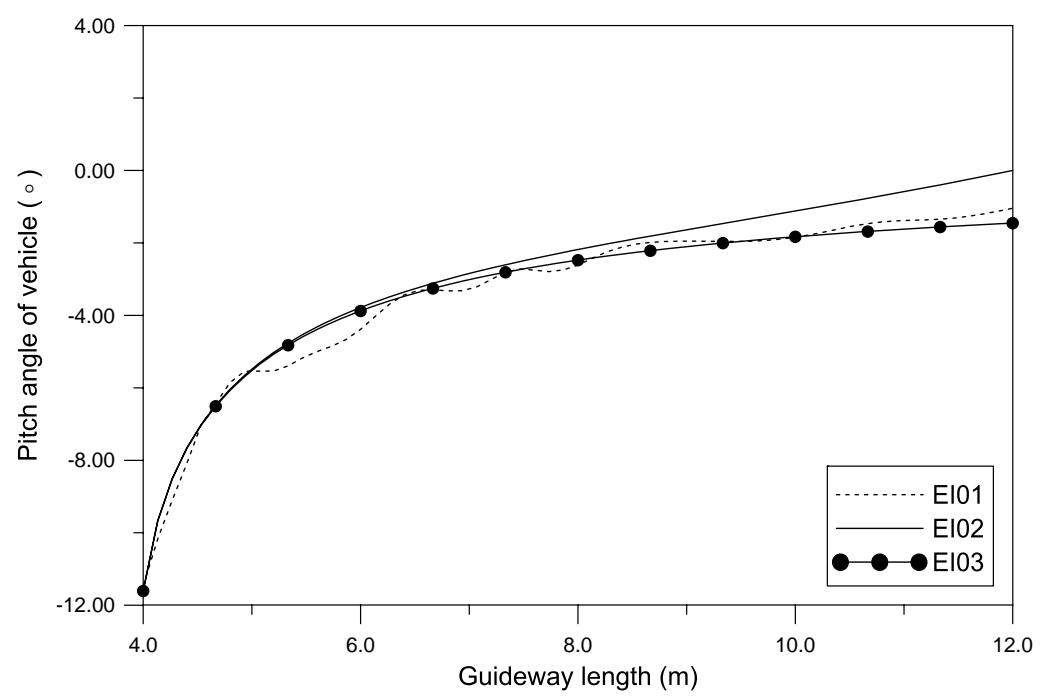

Fig. 13. Effect of length of guideway on pitch angle of vehicle at take-off $\left(\theta-L_{g}\right.$ diagram).

than those obtained using the other two combinations of flexural rigidity, depending on the length of the guideway. Consequently, the results imply that the vehicle should be designed to the maximum extent possible stiffer than the guideway.

\subsection{Influence of distance between shoes of vehicle}

As stated in the previous section, the value of $t_{R}-t_{F}$ significantly affects the tip-off response. The distance between the shoes of the vehicle is a design factor that

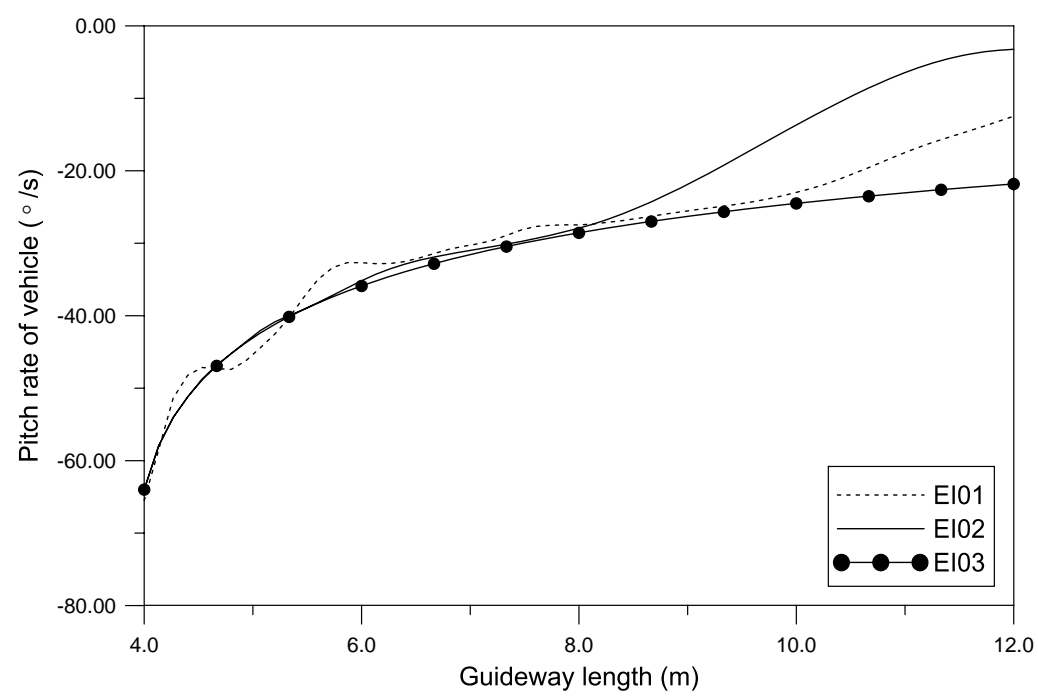

Fig. 14. Effect of length of guideway on pitch rate of vehicle at take-off $\left(\dot{\theta}-L_{g}\right.$ diagram). 


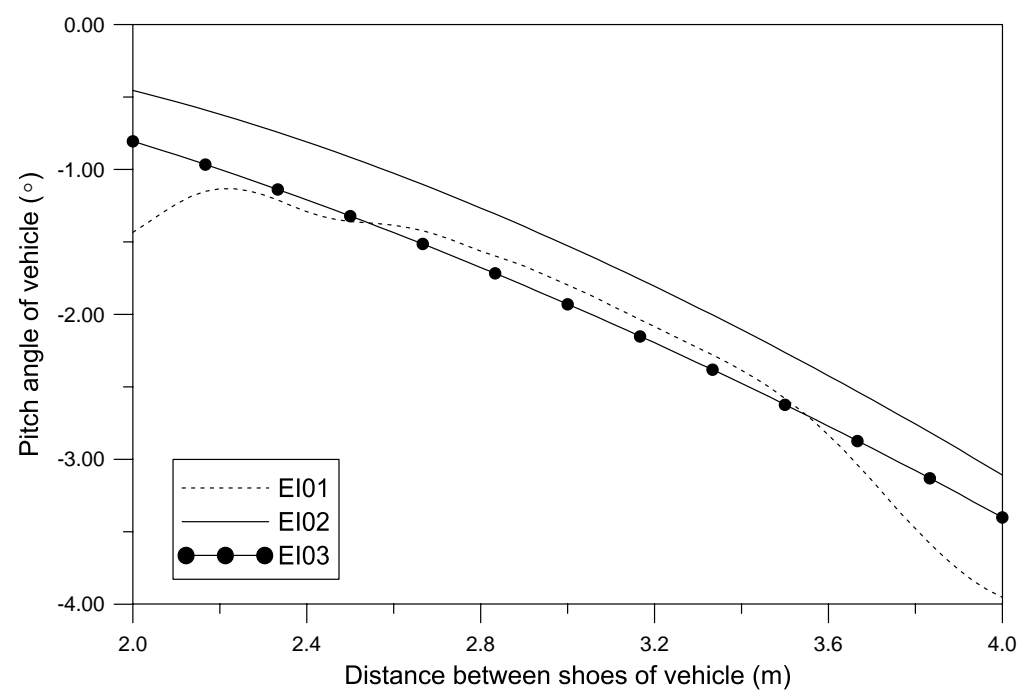

Fig. 15. Effect of distance between shoes of vehicle on pitch angle of vehicle at take-off.

critically influences $t_{R}-t_{F}$. Hence, it is worth showing the variations of pitch angle and pitch rate of the vehicle at take-off with the distance between the shoes of the vehicle. The three combinations of flexural rigidities of the vehicle and the guideway in Table 2 are also considered here.

Figures 15 and 16, respectively, show the variations of the pitch angle and pitch rate of the vehicle at take-off with the distance between the shoes of the vehicle from 2 to $4 \mathrm{~m}$. As expected, as the distance between the shoes of the vehicle increases, the

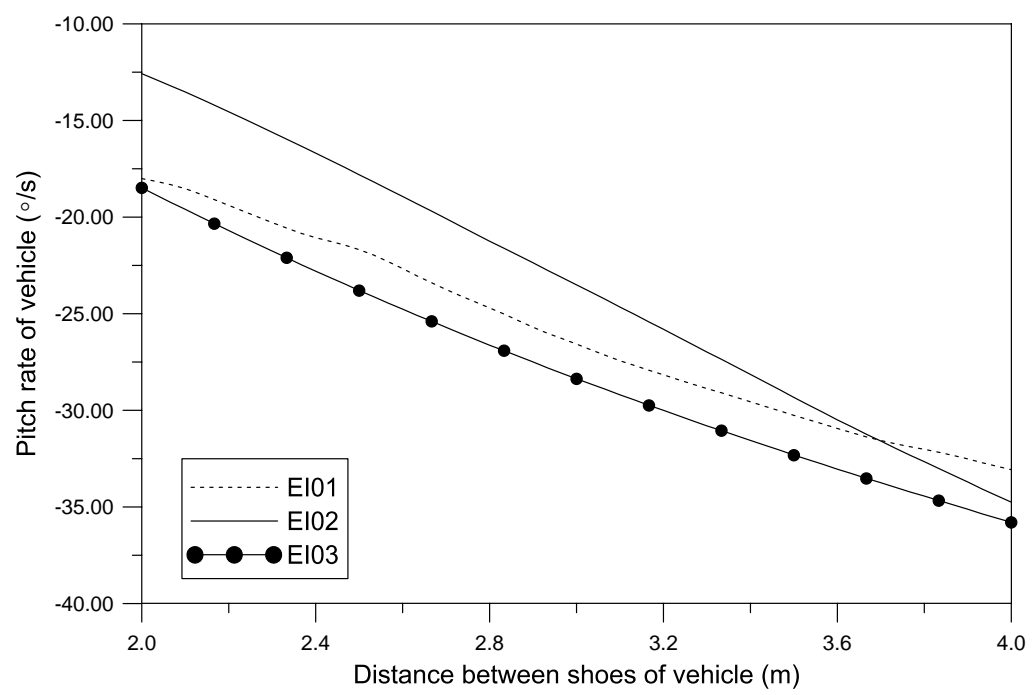

Fig. 16. Effect of distance between shoes of vehicle on pitch rate of vehicle at take-off. 
magnitudes of the pitch angle and pitch rate of the vehicle at take-off increases. Again, the vehicle and guideway with the flexural rigidity combination EI02 always gives a smaller pitch angle and pitch rate for the vehicle than combination EI03. Combination EI01 yields a larger pitch angle than combination EI02.

\subsection{Influence of mass ratio and flexural rigidity ratio}

The mass ratio $M_{r}$ and flexural rigidity ratio $R_{r}$ can be designed for various real applications. The effects of these two ratios on the pitch angle and pitch rate of the

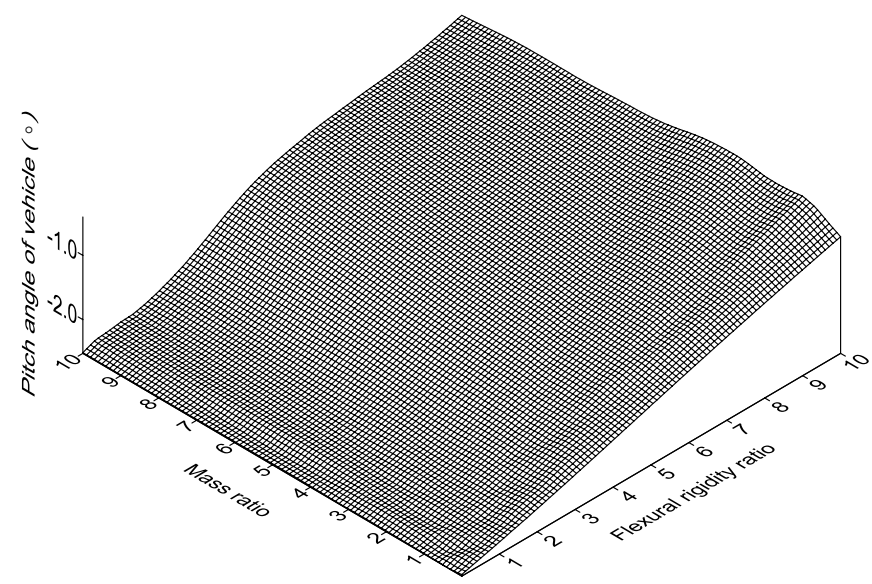

(a)

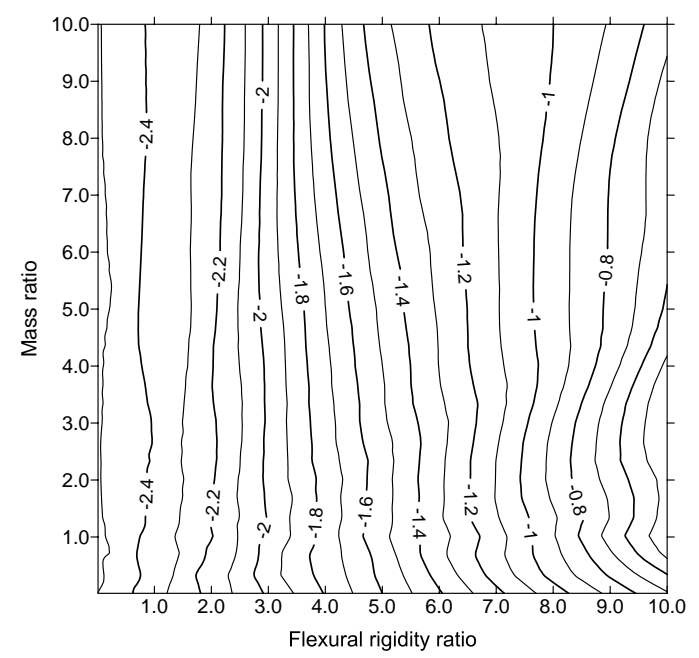

(b)

Fig. 17. Effect of mass ratio and flexural rigidity ratio on pitch angle of vehicle: (a) 3D plot (b) contour plot. 
vehicle at take-off are of interest. These two ratios are changed herein by varying the mass and flexural rigidity, respectively, of the guideway only.

Figures 17 and 18 show the variations of the pitch angle and pitch rate of the vehicle at take-off with $M_{r}$ and $R_{r}$, respectively, in terms of three-dimensional plots and contours. Figure 17 reveals that the pitch angle decreases as $R_{r}$ increases, but a change in $M_{r}$ has no significant effect. Figure 18 indicates that as both $R_{r}$ and $M_{r}$

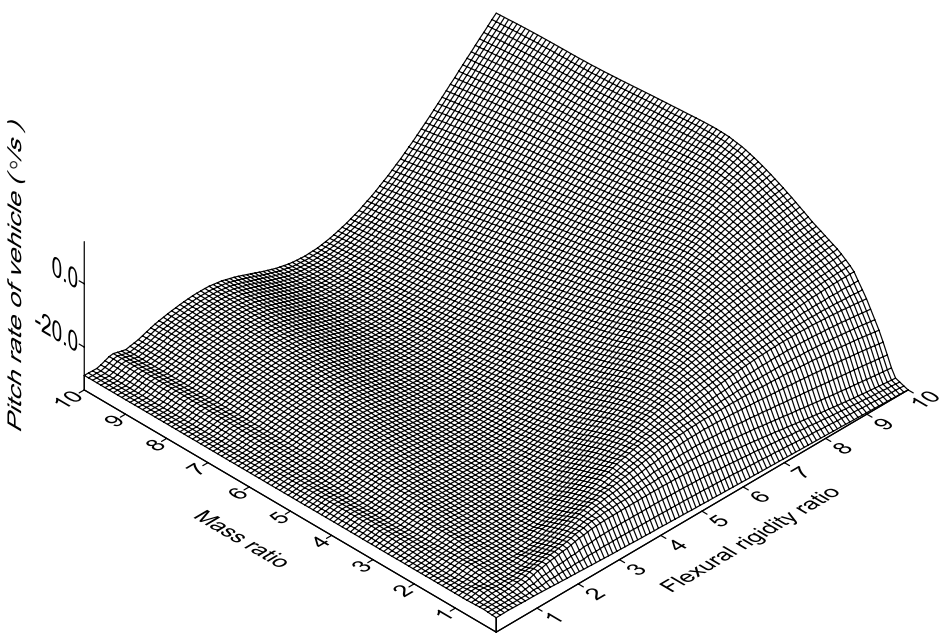

(a)

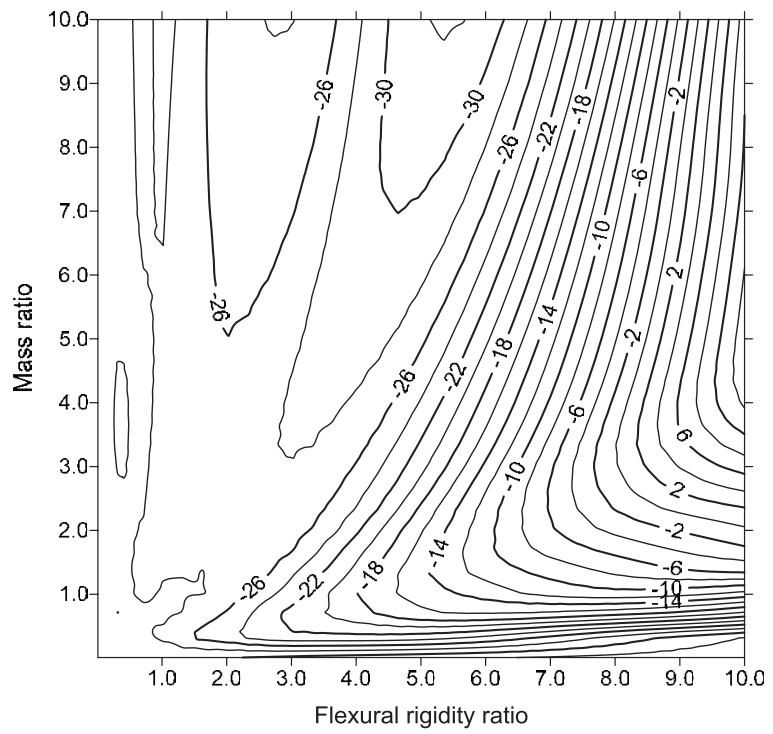

(b)

Fig. 18. Effect of mass ratio and flexural rigidity ratio on pitch rate of vehicle: (a) 3D plot (b) contour plot. 
increase, the pitch rate of the vehicle at take-off decreases. The contour plots are useful for selecting a set of optimum parameters for the launch system.

\section{Conclusions}

In this paper, the governing equations for the dynamic response of a vehicle moving along its guideway was developed based on the Lagrangian approach and mode superposition method. The vehicle and guideway were modeled as beams. In the governing equations, the inertia, Coriolis and centrifugal forces induced by the vehicle, as well as the interaction between the vehicle and guideway, are all taken into account. In the form of nonlinear ordinary differential equations, they are solved using the Petzold-Gear BDF method. Theoretically, the model of an elastic vehicle moving over an elastic guideway, as presented in this paper, captures more closely the practical reality than the commonly used models involving a rigid vehicle or a rigid guideway. The solutions presented herein were validated through the convergence studies using various numbers of modes and time increments and by comparing them with published results for the special cases of a rigid vehicle or a rigid guideway.

The solutions were further employed to investigate the effects of the length of the guideway, the distance between the shoes of the vehicle, the mass ratio and the flexural rigidity ratio of the vehicle to the guideway on the pitch angle and pitch rate of the vehicle at take-off. The numerical results in this study reveal several facts that are useful to the design of a launch system, listed as follows:

- Increasing the length of the guideway reduces the pitch angle and pitch rate of the vehicle at take-off. Reducing the distance between the shoes of the vehicle has a similar effect.

- Increasing the flexural rigidity ratio of the vehicle to the guideway also reduces the pitch angle and pitch rate of the vehicle at take-off, while increasing only the mass ratio significantly reduces the pitch rate.

- The models of elastic vehicle and elastic guideway, rigid vehicle and elastic guideway, and rigid vehicle and rigid guideway yield significantly different pitch angle and pitch rate for the vehicle at take-off, which are crucial to controlling the trajectory of the vehicle after it leaves the guideway. Theoretically, the proposed model captures more accurately the reality of practical applications than the other two models do.

\section{References}

1. Subcommittee on Vibration Problems Associated with Flexural Members on Transit Systems Committee on Flexural Members of the Committee on Metals of the Structural Division, Dynamics of steel elevated guideways-an overview, J. Struct. Eng. ASCE 111 (9) (1984) 1873-1898.

2. G. G. Stokes, Discussion of a differential equation relating to the breaking of railway bridges, Part 5 (1849) 707-735. 
3. S. P. Timoshenko and D. H. Young, Vibration Problems in Engineering (D. Van Nostrand Co., New York, 1955).

4. R. S. Ayre, G. Ford and L. S. Jacobsen, Transverse vibration of a two-span beam under action of a moving constant force, J. Appl. Mech. 17(1) (1950) 1-12.

-5. N. Sridharan and A. K. Mallik, Numerical analysis of vibration of beams subjected to moving loads, J. Sound Vib. 65(1) (1979) 147-150.

6. K. Henchi, M. Fafard, G. Dhatt and M. Talbot, Dynamic behaviour of multi-span beams under moving loads, J. Sound Vib. 199(1) (1997) 33-50.

7. H. H. Jeffcott, On the vibrations of beams under the action of moving loads, Phil. Magazine Ser. 7 8(48) (1929) 66-97.

8. M. M. Stanišić and J. C. Hardin, On the response of beams to an arbitrary number of concentrated moving masses, J. Franklin Inst. 287(2) (1969) 115-123.

-9. J. E. Akin and M. Mofid, Numerical solution for response of beams with moving mass, J. Struct. Eng. ASCE 115(1) (1989) 120-131.

10. M. Dehestani, M. Mofid and A. Vafai, Investigation of critical influential speed for moving mass problems on beams, Appl. Math. Model. 33(10) (2009) 3885-3895.

11. J. J. Wu, Dynamic analysis of an inclined beam due to moving loads, J. Sound Vib. 288 (1-2) (2005) 107-131.

12. J. M. Biggs, Introduction to Structural Dynamics (McGraw-Hill, New York, N.Y., 1964).

13. A. V. Pesterev and L. A. Bergman, Response of elastic continuum carrying moving linear oscillator, J. Eng. Mech. 123(8) (1997) 878-884.

14. Y. B. Yang and B. H. Lin, Vehicle-bridge interaction analysis by dynamic condensation method, J. Struct. Eng. 121(11) (1995) 1636-1643.

15. Y. B. Yang and Y. S. Wu, A versatile element for analyzing vehicle-bridge interaction response, Eng. Struct. 23(5) (2001) 452-469.

16. E. C. Cojocaru, H. Irschik and H. Gattringer, Dynamic response of an elastic bridge due to a moving elastic beam, Comput. Struct. 82(11-12) (2004) 931-943.

17. T. Zhang and G. T. Zheng, Vibration analysis of an elastic beam subjected to a moving beam with flexible connections, J. Eng. Mech. 136(1) (2010) 120-130.

18. C. R. Yao and P. Zhang, Rocket and Guided Missile Launcher Device Design (Beigin Institute of Technology Press, 1998).

19. S. N. Chou, F. P. Cheng and C. S. Huang, Tip-off effect analysis of a vehicle moving along an inclined guideway by considering dynamic interactions, J. Chinese Inst. Eng. 36(2) (2013) 164-172.

20. C. W. Gear and L. R. Petzold, Ode methods for the solutions of differential/algebraic equations, SIAM J. Numer. Anal. 21(4) (1984) 716-728.

21. S. S. Rao, Vibration of Continuous Systems (John Wiley Sons, Inc., Hoboken, New Jersey, 2007).

22. R. W. Clough and J. Penzien, Dynamics of Structures (Computers and Structures, Inc., Berkeley, 2003). 